NIST Special Publication 1265

\title{
Sketchnoting Science \\ How to Make Sketchnotes from Technical Content
}

Rob Dimeo

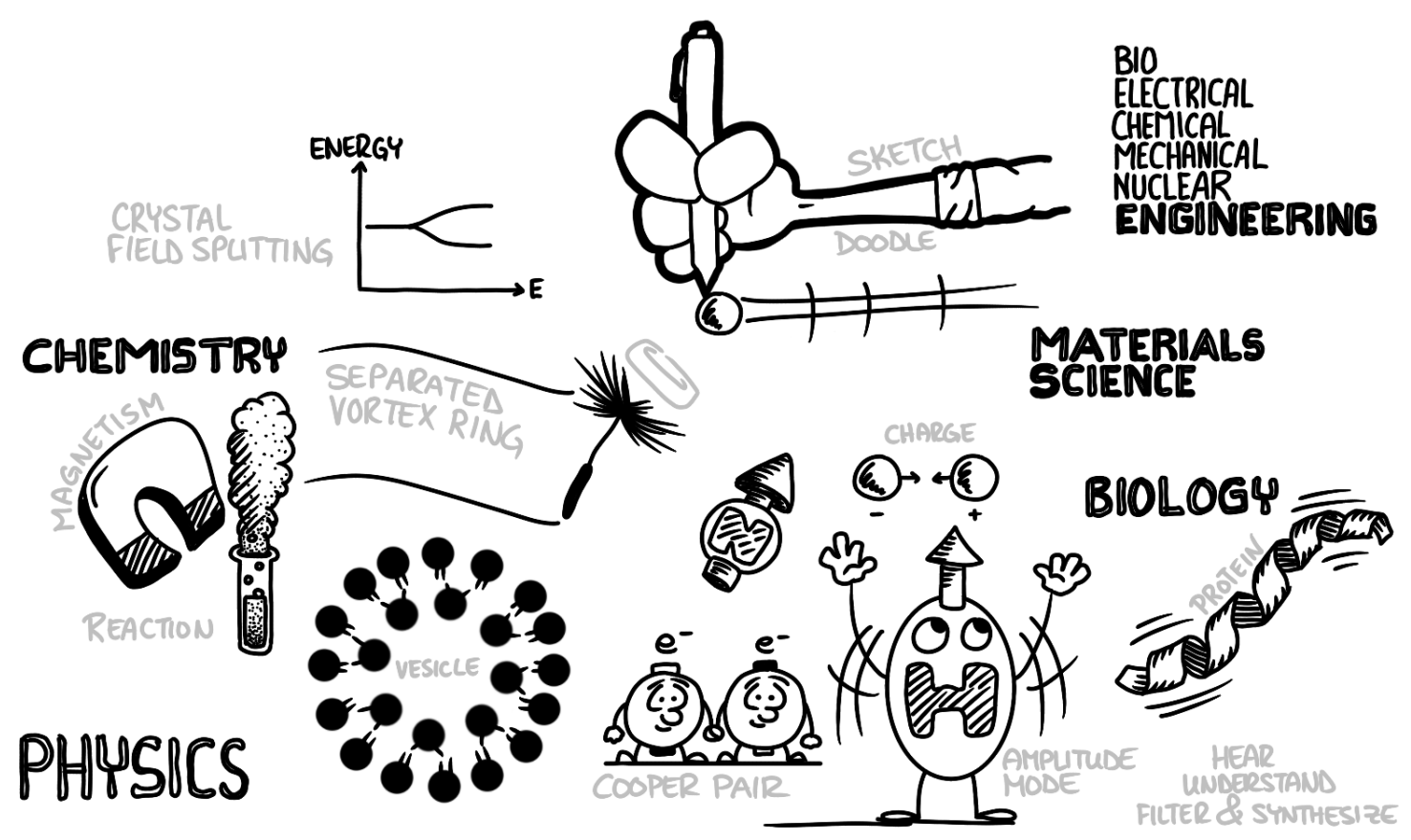

This publication is available free of charge from: https://doi.org/10.6028/NIST.SP.1265

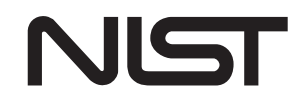

National Instifute of Standards and Technology

U.S. Department of Commerce 


\section{Sketchnoting Science How to Make Sketchnotes from Technical Content}

Rob Dimeo

NIST Center for Neutron Research

This publication is available free of charge from:

https://doi.org/10.6028/NIST.SP.1265

May 2021

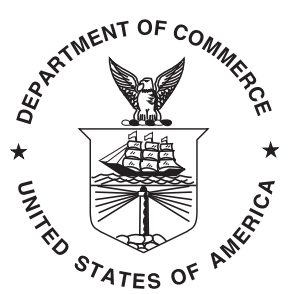

U.S. Department of Commerce

Gina M. Raimondo, Secretary

National Institute of Standards and Technology James K. Olthoff, Performing the Non-Exclusive Functions and Duties of the Under Secretary of Commerce for Standards and Technology \& Director, National Institute of Standards and Technology 
Certain commercial entities, equipment, or materials may be identified in this document in order to describe an experimental procedure or concept adequately. Such identification is not intended to imply recommendation or endorsement by the National Institute of Standards and Technology, nor is it intended to imply that the entities, materials, or equipment are necessarily the best available for the purpose.

National Institute of Standards and Technology Special Publication 1265

Natl. Inst. Stand. Technol. Spec. Publ. 1265, 51 pages (May 2021)

CODEN: NSPUE2

This publication is available free of charge from: https://doi.org/10.6028/NIST.SP.1265 



\section{Foreword}

The Science of Sketchnoting is a book born out of years of practice, crafted by a master whose dedication to sketchnoting is never in question.

I know, because I have watched Rob craft this content over years and years of real-world practice, in his professional life at NIST and in his personal life as well.

I first met Rob in Philadelphia, at a workshop I was teaching at Drexel University. His dedication was clear when I saw that he drove up from Maryland for the weekend of sketchnoting together. I immediately I felt a kinship and brotherhood with Rob.

He is dedicated to not only the craft of sketchnoting and furthering the community by physically becoming part of it, but in learning of Rob's origin story with sketchnoting, I knew he was putting in the time to get better and teach others.

In the years since our first meeting, Rob has continually driven forward his own practice and has ignited the practice of many other sketchnoters, many of them leading scientists.

This book is the encapsulation of Rob's experiences, from the first 30 day challenge he set for himself to sketchnote every meeting, to attending international sketchnote events, and teaching people in person and online, sketchnoting as a practice.

Rob's book is detailed and real. It gives you, the reader, a huge jump-start in your own science sketchnoting practice, based on the hard lessons Rob has learned over the last 6 years.

Keep this book by your side as you progress, because I know you'll read all the way through and then keep it near for reference as you work on your own craft of sketchnoting science.

Mike Rohde

Author of The Sketchnote Handbook[1] and The Sketchnote Workbook[2]

September 2020 



\title{
Preface
}

\author{
"You can do anything with words and pictures!" \\ - Harvey Pekar, American Splendor[3]
}

We are inundated with information every day. Our success depends on how well we process that flood of information: How we analyze it, make decisions with it, and how well we can communicate it. So, it may be somewhat surprising that in the information age, an analog tool incorporating hand-drawn images and handwritten text into a coherent visual representation of content has become such a popular mode of information recording. This tool is called sketchnoting. Sketchnotes is the term that Mike Rohde invented to describe a form of notes that incorporates simple drawings and hand-drawn typography.[1]

I first learned about sketchnotes in 2014 when I was looking for tools to help broaden my knowledge of materials science and deepen my understanding in areas of science that were outside of my chosen field of study. I learned how to create my own sketchnotes initially based on non-technical content: discussions at work meetings, administrative information, and TED talks. ${ }^{1}$ I learned that making sketchnotes from technical content was much more difficult and time-consuming because it takes time to understand the technical content, process the information in a manner suitable to summarize visually, and draw the sketchnote. I find the first two steps to be more time consuming for technical sketchnotes than for those based on non-technical content.

Over time I developed a workflow for creating sketchnotes based on technical content. I applied this workflow to content from scientific papers and technical presentations. I put it into practice and it has made a major difference. The process of creating these technical sketchnotes has enhanced my understanding of a broad range of topics, increased my retention and recall of specific areas of research, and even improved my ability to communicate technical material. I have built up a significant library of scientific sketchnotes which are important references for me.

In this book I introduce you to an approach for making sketchnotes based on technical content. The reader is assumed to have some technical background, though not in a specific scientific discipline. This will become clear when the exercises are introduced. They are based on scientific material from the physical sciences which the reader is expected to be able to understand to some degree, even if he/she is not an expert in the field from which the example originates. The reader is assumed to have some experience making

\footnotetext{
${ }^{1}$ https://ted.com
} 


\section{Preface}

his/her own sketchnotes based on non-technical subject matter. I provide a short summary of sketchnotes and the motivation for sketchnoting scientific material in the first chapter. In chapter two, the focus is on how to create your own sketchnotes based on written technical content. In chapter three, we extend the approach of chapter two to creating sketchnotes of content based on technical presentations. In chapter four I show a different application of sketchnotes: how to use a sketchnote as the main visual for a technical presentation. Chapter five includes a selection of my scientific sketchnotes. I put them in chronological order so you can see the progression over time. From the first one I made based on a seminar in 2014 to a very recent seminar in 2020, you can see how my style and organization of content evolved. Sketchnoting is a skill composed of active listening, summarizing a complicated message, and rendering quick sketches to represent abstract concepts. It takes time to develop a skill and improvement comes from deliberate practice. Sketchnoting technical content is difficult and, like other challenging things, I believe that it is worthwhile.

The reader should regard the exercises throughout this book as essential. Since sketchnoting is a skill, it requires practice. Likewise, sketchnoting scientific content is an extension of that skill and the exercises in this book have been designed to carry the reader from being comfortable sketchnoting non-technical content to making sketchnotes of technical material. Therefore, it is essential that the reader performs these exercises if he/she is to achieve the overall goal of learning how to sketchnote from various sources of technical content. This book accompanies a sketchnote workshop that I offer at NIST where attendees learn how to make sketchnotes from technical content. Several exercises from this book form the basis for the in-class workshop activities. In particular, Exercises 2.1.1, 2.2.1, and 3.4.1, which are related to the collective motion of humans in mosh pits[4], carry the reader from preparing to sketchnote a presentation, sketchnoting a technical publication, and finally to actually sketchnoting a technical presentation. In my opinion, the research described in the referenced publication and video in those examples is accessible and, therefore, is useful for learning how to apply the methods in this book.

It is a great pleasure to acknowledge the help and encouragement I have received from numerous scientists and sketchnoters from around the world. First, Mike Rohde, the Sketchnote Guy and a good friend, who gave me valuable feedback on an early draft of this manuscript. I learned how to sketchnote from his wonderful hand-drawn book, The Sketchnote Handbook.[1] I enjoyed our many discussions about how best to present sketchnote material in workshops and how to nurture a community. His boundless generosity has built a global community of welcoming practitioners. I owe thanks to my friend and fellow sketchnoter, Steve Silbert, who graciously agreed to edit this book. I thank Dr. William Ratcliff, a talented physicist and a sketchnoter of highly technical scientific material. He was extremely generous with his time, providing valuable feedback on the content of this book. I also thank Dr. Jamie Weaver, an analytical chemist and practiced sketchnoter, for reading the manuscript and providing helpful feedback. In addition, I owe visual thinker Wendi Pillars a debt of gratitude for reviewing an early draft of this manuscript and providing valuable feedback, especially on the pedagogical aspects. I am grateful to the growing community of science sketchnoters including Katharina Theis-Bröhl, Mathis Riehle, and 
Franziska Schwarz who have shared their passion for science sketchnotes and from whom I've learned much. Katharina was an especially strong influence for me as she was a very enthusiastic and early practitioner of science sketchnoting. I learned much from our many lengthy discussions focused on science sketchnotes and I have enjoyed her creative approach to this difficult skill. Practitioners in the growing field of science comics such as Jay Hosler and Nick Sousanis have also influenced my thoughts on effective ways to visualize and communicate scientific material. Even though my sketchnotes are not necessarily meant as a mechanism to communicate science to a broad audience, the aesthetic sensibilities of science comics have had an impact on how I construct a page. I also extend thanks to the entire staff of the NIST Center for Neutron Research (NCNR) who unwittingly participated in my visual communication experiment for the last 6 years. I can only guess that the presence of sketchnotes above a few desks and on a couple doors at the NCNR indicates some acceptance of this as a means of scientific communication and/or reference. I also thank Dr. Willie May, former Director of NIST, who "caught" me sketchnoting in a leadership meeting once and did not hesitate to voice approval. In fact, I have been delighted that so many senior leaders at NIST have been accepting of this. I'd like to acknowledge the support of all education and outreach activities at the NCNR by the Center for High Resolution Neutron Scattering, a partnership between the National Institute of Standards and Technology and the National Science Foundation under Agreement No. DMR-2010792. CHRNS supported many high school and undergraduate students who participated in several sketchnote workshops. Finally, I thank the NIST sketchnote community who attended my sketchnote workshops over the years. They have embraced the workshop material with enthusiasm, given great feedback, shown me numerous ways to visualize abstract concepts, and I've learned much from them.

Rob Dimeo

Gaithersburg, MD

January 13, 2021 



\begin{abstract}
A method of incorporating hand-drawn visuals into notes on technical topics from scientific publications and presentations is described in this book. In the introductory chapter, sketchnotes are defined and the advantages of making sketchnotes based on technical content is presented. Chapter 2 leads the reader through the process of making sketchnotes from written content, including scientific publications. Chapter 3 presents an approach to making sketchnotes from presentations with a detailed example from a scientific seminar available via an online video. Many exercises are provided in chapters 2 and 3 for the reader to practice the methods. Chapter 4 describes an alternative application: how to make and present sketchnote visuals for technical presentations. The final chapter gives several specific examples of sketchnotes based on scientific papers and technical presentations.
\end{abstract}

\title{
Key words
}

education; learning; notes; presentations; science; sketchnotes; visuals; visual notes. 


\section{Table of Contents}

Foreword $i$

Preface $\quad$ iii

Abstract vii

1 Sketchnotes 1

1.1 What are sketchnotes? 1

1.2 Why make sketchnotes? 3

1.3 Why I make scientific sketchnotes 3

1.4 Tools for making sketchnotes 4

2 Written Technical Content 5

2.1 Sketchnoting a title \& abstract 5

2.2 Sketchnoting a scientific paper 9

3 Technical Presentations 17

3.1 Preparation 18

$\begin{array}{ll}3.2 & \text { During the talk }\end{array}$

$\begin{array}{ll}3.3 \text { After the talk } & 19\end{array}$

3.4 Example: Sketchnoting a live talk 22

4 Presentation Planning \& Visuals 27

$\begin{array}{ll}4.1 & \text { Planning tool } \\ & 27\end{array}$

$\begin{array}{lll}4.2 & \text { Presentation visual } & 27\end{array}$

5 Selected Science Sketchnotes 35

6 References $\quad 49$ 


\title{
1. Sketchnotes
}

\author{
"Ideas, not art!"
}

- Mike Rohde, The Sketchnote Handbook[1]

\subsection{What are sketchnotes?}

Mike Rohde defines sketchnotes ${ }^{1}$ as "rich visual notes created from a mix of handwriting, drawings, hand-drawn typography, shapes, and visual elements like arrows, boxes, and lines.”[1, p.2] An example of a sketchnote is shown in Figure 1.1. Arrows, lines, containers, hand-drawn typography, drawings, and data sketches are all present.

At first glance this sketchnote might resemble an infographic. While an infographic is intended to convey information quickly and clearly to a broad audience, a sketchnote has an audience of one: the one who created the sketchnote (i.e. you, the sketchnoter). Your sketchnote need not be meaningful to anyone but you. It is based on your knowledge and it is a reference that will be most meaningful to you. For one without specialist knowledge of quantum phenomena, spin dynamics, and neutron scattering, it is unlikely that the sketchnote that I drew in Figure 1.1 will have much meaning.

The elements and structure of the sketchnote in Figure 1.1 should be familiar to you. The title is written with red ink and uses a combination of printing and script. The mixed type and the different text color are ways of achieving contrast, and contrast is an effective way to get attention. The downward vertical flow of information is influenced heavily by the portrait orientation of the page. Three large, hand-drawn rectangles form the major containers of the primary elements of the presentation and two cloud containers delineate simple graphical representations of different phases of water and compare them with the different phases of a system of spins. The drawings are simple. The drawing of the magnet with a ket vector (familiar to practitioners of quantum mechanics) in a liquid puddle is a cartoon representation of a quantum spin liquid. Cartoons are not necessary in sketchnotes, but I often include them. I find cartoons to be quick, effective and visually appealing. In this cartoon, the concrete (water) is related to the abstract (spins). The connection of the concrete to the abstract is a key ingredient of a good talk and shows that the speaker cares

\footnotetext{
${ }^{1}$ Though the word sketchnote is not in the Merriam-Webster dictionary ${ }^{2}$, it will be used in various forms in this book. It can be used as a noun: e.g., ...making a sketchnote. It can be used as a verb: e.g., ...sketchnoting a talk. Sketchnoter refers to someone who makes a sketchnote.
} 


\section{Sketchnotes}

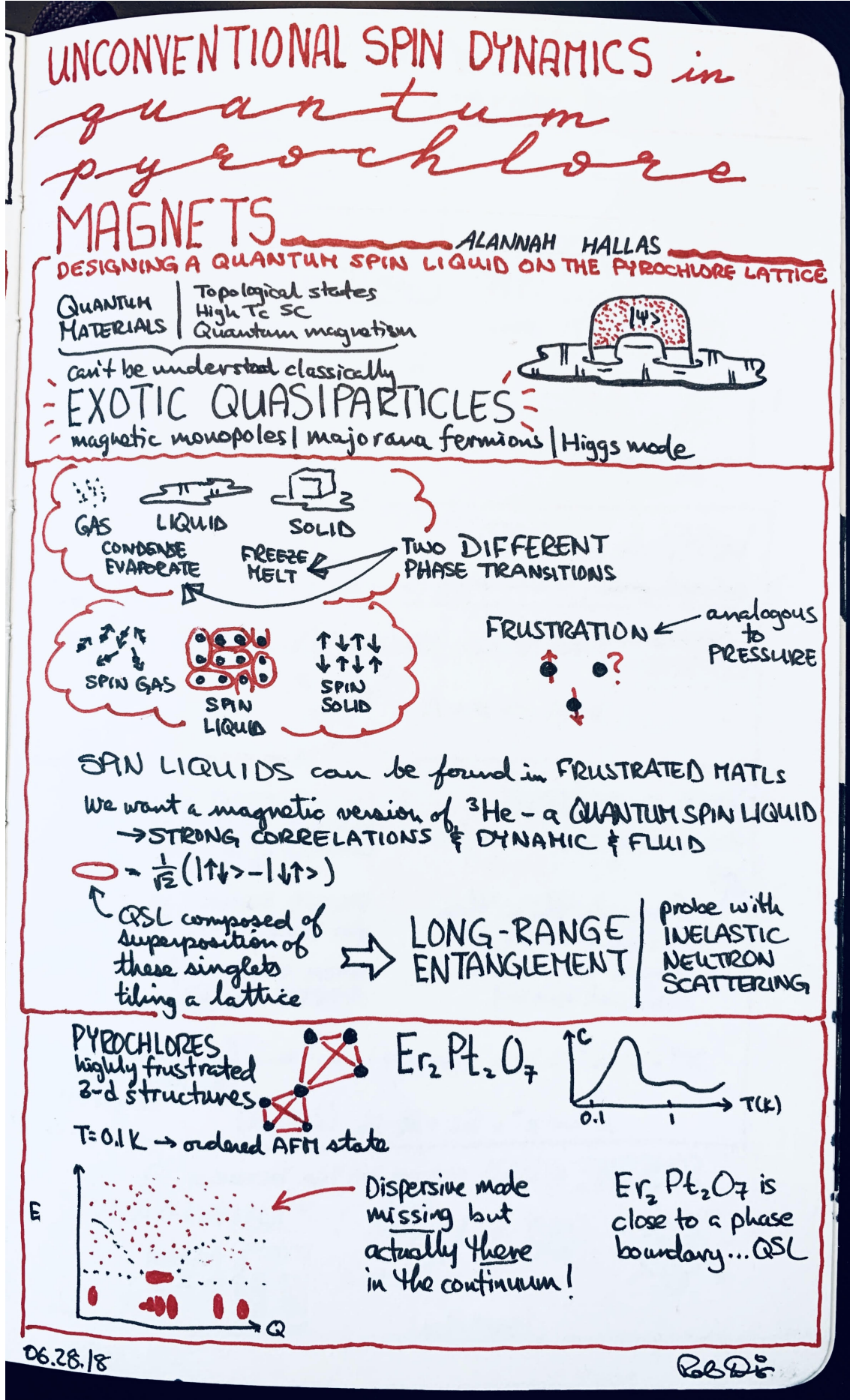

Fig. 1.1. Sketchnote based on a presentation by Alannah Hallas at the American Conference on Neutron Scattering, June 2018. 
that the audience can relate to an esoteric subject. Sketching such analogies is very helpful in scientific sketchnotes.

One other important aspect of Figure 1.1 is that it is not composed of too many complete sentences or even many sentence fragments. It has a few summary statements and several drawings and data sketches. Sketchnotes are meant to capture the big ideas. In a sketchnote, the big ideas are evident in the hierarchy, via the larger, bolder lettering and content in containers. Sketchnotes are not meant to provide a complete recording of content. Sketchnotes are focused on big ideas. Moreover, the drawings are often simple and drawn quickly. Sketchnotes need not incorporate polished drawings that one might find in an infographic created by a professional illustrator. Mike Rohde summarizes sketchnotes succinctly as about "ideas, not art!"[1, p.17]

\subsection{Why make sketchnotes?}

There are several reasons why you should make sketchnotes. In her book, The Doodle Revolution[5], Sunni Brown states that visual notetaking leads to a high level of comprehension, and increases retention and recall. The combination of simple visuals with words is analogous to glance media such as a billboard, designed to capture attention and leave a quick impression. Sketchnotes can have more visual appeal than conventional notes since they contain sketches and words. This appeal comes from the fact that they capture the main ideas in a visual hierarchy rather than the smallest details. A sketchnote's visual hierarchy and minimum of details means that you will not get bogged down trying to tease out the main ideas as you might from a page of conventional handwritten notes. This is a key reason for their utility. You may be more inclined to look at your sketchnotes more often than reviewing your conventional notes.[1]

The power of sketchnotes comes from the act of sketchnoting and the appeal of the resulting visual artifact. The act of sketchnoting often requires more concentration than conventional notetaking. When you are making a sketchnote from a presentation, for example, the act of sketchnoting requires you to listen to the speaker, look at the visuals, process the content (analyze \& summarize), and sketch simultaneously. These are difficult skills on their own and even more challenging to do simultaneously. The higher degree of concentration, writing words by hand, and sketching can provide a deeper connection with the material, enhancing retention and recall, than passive listening or conventional notetaking.[6] The resulting artifact — the sketchnote itself-can be more pleasurable to view than conventional notes. This leads to high "re-read value" and repeated views of the sketchnote can lead to increased retention and recall.

\subsection{Why I make scientific sketchnotes}

I started sketchnoting for a simple reason. I wanted to broaden my knowledge of science and deepen my understanding in areas of science that were outside of my own research area. There are several possible ways to achieve this: take courses in different disciplines (or get additional degrees in multiple disciplines), read textbooks, attend technical presentations 


\section{Sketchnotes}

by experts in the field, attend presentations by technical experts that are targeted to nonexperts, and/or read publications from the peer-reviewed scientific literature. Attending more college might not be a realistic choice for everyone ${ }^{3}$ and the other possibilities listed above are typically more accessible to practicing scientists and engineers.

Regardless of the source of technical content, I would often take notes as a part of learning. Unfortunately, my notes were of little use. There were key ingredients missing. First, I seldom looked at them. Often they consisted of a set of unrelated and incomplete sentences, a few orphaned equations, and incomprehensible figures with no annotations. My notes all followed the chronological sequence of the presentation. There there were no distinguishing structural characteristics that drew attention to key concepts. Second, there were no memorable cues in my notes that would help me remember anything from the content. My notes appeared bland and nothing drew me in to look at them again. Third, taking notes for me was dull and uninteresting work. If taking notes is drudgery and I wouldn't look at them again, why bother?

Sketchnotes offered an attractive alternative. A sketchnote is a brief, visual summary of that content. Like an abstract in a peer-reviewed science publication, a science sketchnote is a summary description. Unlike an abstract, a science sketchnote contains both words and sketches. Unlike an abstract, a science sketchnote is targeted to you, the sketchnoter, and assumes your scientific knowledge and experience. Thus, the science sketchnote that you make is a visual reference that can have long-lasting meaning for you.

Based on my experience making these technical sketchnotes, the process can enhance your understanding of a broad range of topics, increase your retention and recall of specific areas of research, and improve your ability to communicate technical material. When you make it a regular practice to make sketchnotes of science articles and presentations, you will build up your own library of sketchnotes which will be great references for you.

\subsection{Tools for making sketchnotes}

Making sketchnotes requires only a sheet of paper and a pen but you can also use digital tools such as a tablet, stylus and drawing application. There has been much written about tools for sketchnotes so I will not address those here.[7, pp.19-28] For scientific sketchnotes I occasionally use a sketchbook and a black gel pen. Most of my science sketchnotes were made on an iPad Pro with an Apple Pencil using the Procreate art application by Savage Interactive. ${ }^{4}$

\footnotetext{
${ }^{3}$ If you are a science or engineering student, sketchnoting may be a useful skill to learn.

${ }^{4}$ Any mention of commercial products is for information only; it does not imply recommendation or endorsement by NIST.
} 


\section{Written Technical Content}

"Nothing makes you feel stupid quite like reading a scientific journal article."

- Adam Ruben, How to read a scientific article[8]

Sketchnotes you make based on technical publications can be valuable references for you. As described in Section 1.1, their benefits are derived from the act of their creation and having them as one-page references you can review. Creating them can even help prepare you for upcoming talks that you wish to sketchnote.

Making sketchnotes from written content in a specific technical discipline can be timeconsuming and challenging, even if you are an expert in that field. When reading a publication you should be able to extract the research question being asked, its importance, the approach to answer that question, and what conclusions can be drawn from executing that approach. Much of this information should be available in the title and abstract. These are items you should consider including in your sketchnote. However, several things can make this difficult, including that the research topic (or certain aspects of the research such as the experimental approach) might be unfamiliar to you. As we will see in this chapter, the way to overcome this is with preparation and research. Sketchnoting a publication has the advantage of no time pressure. You can pause anytime when reading the paper to research unfamiliar terms or concepts.

We will approach this in two steps. First, we will create sketchnotes based just on the title and abstract of a scientific publication. Next, we will extend this to an entire scientific publication.

\subsection{Sketchnoting a title $\&$ abstract}

A well-written abstract for a scientific publication contains a summary of the research performed, its importance, the methods used, and key results. This is enough information for you to create a sketchnote. As an example, consider the title and abstract from a paper by Albert Einstein listed below.[9] To keep things simple, draw your sketchnote with only one sketch and one phrase and as many labels as you wish. ${ }^{1}$ My sketchnote of the abstract is shown in Figure 2.1.

\footnotetext{
${ }^{1}$ It is a useful exercise to impose constraints like these to hone your listening skills during presentations.
} 


\section{Written Technical Content}

On the Movement of Small Particles Suspended in a Stationary Liquid Demanded by the Molecular-Kinetic Theory of Heat

In this paper it will be shown that according to the molecular-kinetic theory of heat, bodies of microscopically-visible size suspended in a liquid will perform movements of such magnitude that they can be easily observed in a microscope, on account of the molecular motions of heat. It is possible that the movements to be discussed here are identical with the so-called "Brownian molecular motion"; however, the information available to me regarding the latter is so lacking in precision, that I can form no judgment in the matter (I).

If the movement discussed here can actually be observed (together with the laws relating to it that one would expect to find), then classical thermodynamics can no longer be looked upon as applicable with precision to bodies even of dimensions distinguishable in a microscope : an exact determination of actual atomic dimensions becomes possible. On the other hand, had the prediction of this movement proved to be incorrect, a weighty argument would be provided against the molecular-kinetic conception of heat.
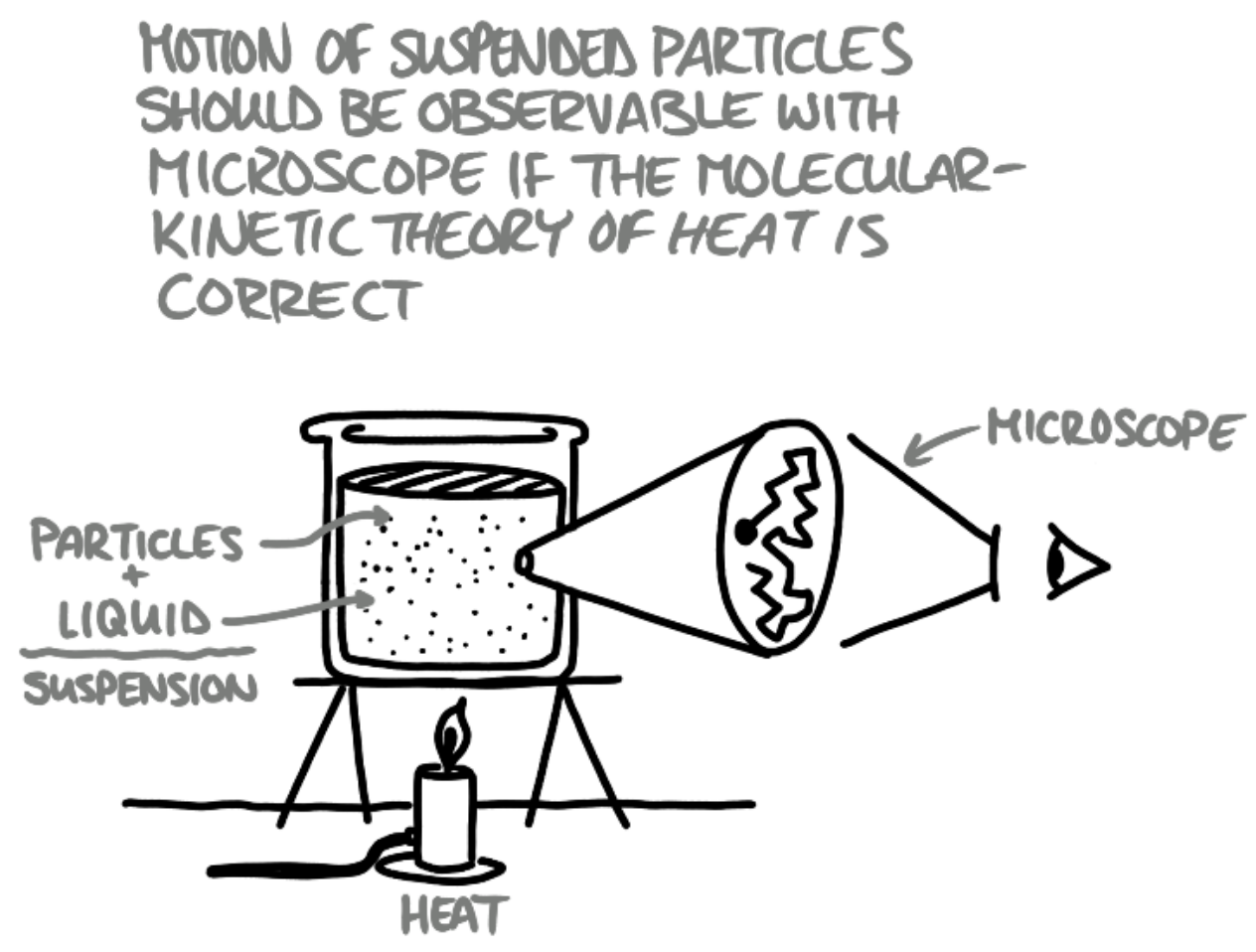

Fig. 2.1. Sketchnote of the title and abstract from paper by Albert Einstein.

There are several things to keep in mind as you look at Figure 2.1. First, I chose to use a descriptive phrase to characterize the main point of the abstract, rather than using the 
full title. The title would have been an acceptable choice, but I wanted to focus on the conclusion of the paper as described in the abstract. If I were to use this sketchnote as a reference then I would include the article's citation. However, this is a warm-up exercise, so it is unnecessary.

Second, I chose to draw an experimental setup that represented how the experiment proposed by Einstein might be performed. I drew a suspension (defined as particles suspended in a liquid... or dots floating in a volume) contained in a beaker that is being heated from below. The particles are proposed to move around so that a microscope (crudely drawn next to the eye) is all that would be needed to observe these motions. Note that I labeled the microscope. This is not a necessary reminder that the eye is looking through the microscope since the summary phrase suggests use of a microscope. One thing that is NOT included in the abstract is a definitive statement that this motion is Brownian motion. I drew a single particle moving erratically, appearing to change direction abruptly, to represent the Brownian movement of a particle based on my background. Nor have I defined the molecular-kinetic theory of heat. If I was unfamiliar with this concept, then I might include it somewhere in the sketchnote.

Finally, several additional annotations are included in the sketch. These labels weren't necessary because I can identify these elements without them. However, I often err on the side of adding more information in my scientific sketchnotes. I should also note there is little distinction from a well-annotated figure and a sketchnote in this case. This is by the design of the exercise and the constraints imposed. Your sketchnote is not likely to appear exactly like mine. Your experience, assumptions and drawing style affects the type of sketch, the annotations, the words you use in your annotations, and even the complexity of your sketchnote. Your sketchnote might be simpler or more elaborate. The important thing to remember is that the sketchnote is for you. You want to be able to review this in the future and understand the big ideas that were stated in the abstract.

This type of exercise, though simple to pose, is not easy to do. It may be even extraordinarily difficult if the content is too far removed from your background. You must spend enough time reading the title/abstract and processing the information so that you can describe it visually. You may have to do some additional research on terms or concepts that you don't understand before you can fully appreciate the abstract. Though I had some familiarity with the content in the previous example, I read the abstract several times and thought about it at length before I drew the sketchnote.

Now it's your turn to practice turning an abstract into a sketchnote in the following exercises. As you create sketchnotes for these, keep the following points in mind: (1) feel free to use any resources at your disposal including a web search on unfamiliar terms or to seek inspiration for images that you would like to sketch; (2) you need not include everything in the abstract in your sketchnote - remember that sketchnotes are about the big ideas; and, (3) use one sketch, one phrase, and as many annotations as you wish. 
Exercise 2.1.1. Create a sketchnote based on the following title and abstract.

Collective Motion of Humans in Mosh and Circle Pits at Heavy Metal Concerts[4]

Human collective behavior can vary from calm to panicked depending on social context. Using videos publicly available online, we study the highly energized collective motion of attendees at heavy metal concerts. We find these extreme social gatherings generate similarly extreme behaviors: a disordered gaslike state called a mosh pit and an ordered vortexlike state called a circle pit. Both phenomena are reproduced in flocking simulations demonstrating that human collective behavior is consistent with the predictions of simplified models.

Exercise 2.1.2. Create a sketchnote based on the following title and abstract.

\section{In-vivo biomagnetic characterisation of the American cockroach[10]}

We present a quantitative method, utilising a highly sensitive quantum sensor, that extends applicability of magnetorelaxometry to biological samples at physiological temperature. The observed magnetic fields allow for noninvasive determination of physical properties of magnetic materials and their surrounding environment inside the specimen. The method is applied to American cockroaches and reveals magnetic deposits with strikingly different behaviour in alive and dead insects. We discuss consequences of this finding to cockroach magneto-reception. To our knowledge, this work represents the first characterisation of the magnetisation dynamics in live insects and helps to connect results from behavioural experiments on insects in magnetic fields with characterisation of magnetic materials in their corpses.

Exercise 2.1.3. Create a sketchnote based on the following title and abstract.

A separated vortex ring underlies the flight of the dandelion[11]

Wind-dispersed plants have evolved ingenious ways to lift their seeds. The common dandelion uses a bundle of drag-enhancing bristles (the pappus) that helps to keep their seeds aloft. This passive flight mechanism is highly effective, enabling seed dispersal over formidable distances; however, the physics underpinning pappus-mediated flight remains unresolved. Here we visualized the flow around dandelion seeds, uncovering an extraordinary type of vortex. This vortex is a ring of recirculating fluid, which is detached owing to the flow passing through the pappus. We hypothesized that the circular disk-like geometry and the porosity of the pappus are the key design features that enable the formation of the separated vortex ring. The porosity gradient was surveyed using microfabricated disks, and a disk with a similar porosity was found to 
be able to recapitulate the flow behaviour of the pappus. The porosity of the dandelion pappus appears to be tuned precisely to stabilize the vortex, while maximizing aerodynamic loading and minimizing material requirements. The discovery of the separated vortex ring provides evidence of the existence of a new class of fluid behaviour around fluid-immersed bodies that may underlie locomotion, weight reduction and particle retention in biological and manmade structures.

Exercise 2.1.4. Create a sketchnote based on the following title and abstract.

Increasing the size of a piece of popcorn[12]

Popcorn is an extremely popular snack food in the world today. Thermodynamics can be used to analyze how popcorn is produced. By treating the popping mechanism of the corn as a thermodynamic expansion, a method of increasing the volume or size of a kernel of popcorn can be studied. By lowering the pressure surrounding the unpopped kernel, one can use a thermodynamic argument to show that the expanded volume of the kernel when it pops must increase. In this project, a variety of experiments are run to test the qualitative validity of this theory. The results show that there is a significant increase in the average kernel size when the pressure of the surroundings is reduced.

\subsection{Sketchnoting a scientific paper}

Let's extend the approach from the previous section and make a sketchnote based on text from a whole paper. We will go into some detail with a specific example so that you can understand my workflow and see how it was applied to a specific scientific paper. Not surprisingly, the first step to creating a sketchnote based on a scientific publication is to start with the title and abstract. As you have seen, the title and abstract should give you a high-level view of the paper's content. In some cases, you may be able to obtain the main aspects of the sketchnote from the title and abstract and then include additional details from the contents of the paper.

Let's consider the paper by Virot and Ponomarenko titled Popcorn: critical temperature, jump and sound [13]. The abstract for the paper is reproduced below.

Popcorn bursts open, jumps and emits a 'pop' sound in some hundredths of a second. The physical origin of these three observations remains unclear in the literature. We show that the critical temperature $180^{\circ} \mathrm{C}$ at which almost all of popcorn pops is consistent with an elementary pressure vessel scenario. We observe that popcorn jumps with a 'leg' of starch which is compressed on the ground. As a result, popcorn is midway between two categories of moving systems: explosive plants using fracture mechanisms and jumping animals using muscles. By synchronizing video recordings with acoustic recordings, we propose that the familiar 'pop' sound of the popcorn is caused by the release of water vapour. 


\section{Written Technical Content}

We first familiarize ourselves with the goal of the paper by making a sketchnote based on the abstract and title as we did in the previous section. Consider the sketchnote shown in Figure 2.2. Following the same guidelines as in the previous section, a single sketch is my visual interpretation of the burst-jump-pop sequence, along with several annotations and a summary statement. The statement is posed as the main research question. Note that the research question is separated from the sketch by horizontal lines below and above it and centered on the page. This draws attention to it and makes it unambiguous. The sketch is composed of the burst-jump-pop sequence of the popcorn being recorded by synchronized audio and video. The measurement method is represented by the video camera and microphone connected to a laptop computer. In the sketch of the burst-jump-pop sequence, the popcorn is drawn the same way for each step but just repositioned with additional elements sketched around it to change the context. For example, the "burst" phase could have been drawn as an unpopped kernel bursting into the popped piece. Instead, the burst is drawn with arcs drawn on both sides of the popped popcorn. This simple and efficient trick is borrowed from cartoons. The arcs are referred to as emanata, a term invented by cartoonist Mort Walker (Beetle Bailey)[14], and more conventionally referred to as cartoon effects \& accessories by cartoonist Bruce Blitz.[15] These additions can be an effective shorthand to represent complicated phenomena. Other examples of these emanata include the lines drawn underneath the popcorn in the "jump" phase making it appear to jump off the ground, the shadow on the ground underneath it, and the elements drawn around the popcorn piece in the "pop" phase making it appear that it is popping at that moment. One other cartoon feature present in the sketchnote is the shoe added to the "starch leg" on the popcorn piece in the jump phase. This is unnecessary, but I find that adding whimsy to sketchnotes via such cartoon elements adds visual appeal. In general, anthropomorphizing inanimate objects with cartoon elements can be a quick and simple way to add visual interest.

Sketchnoting a title and abstract is not typically an end itself, but part of the process in preparation for making the more detailed sketchnote for the whole paper. Nevertheless, the sketchnote in the figure is a good summary visual representation of the content. We might expect the final sketchnote to have some similar elements to that in Figure 2.2.

The next step in the workflow is reading the paper and viewing the figures for understanding. So that you can best understand the sketchnote development in this section and how I applied my workflow to develop the sketchnote, read the paper Popcorn: critical temperature, jump and sound.[13] Reading a technical paper is much different from reading a magazine article that was written for a general audience. The structure is different, and the content is specialized. Consequently there are different approaches to consuming the information in a scientific paper.[8],[16] It is assumed that you already have a strategy for reading and understanding a scientific paper but you can find good tips in references [8] and[16]. When reading a paper, typically, I make multiple passes through it. In the first pass, I read the title and abstract, then skim the introduction and conclusions, and then skim the figures. When skimming the figures, I look for one that summarizes the answer to the research question posed. If one such figure exists, then I make a special note of it. Some representation of that figure will likely appear in the final sketchnote of the paper. If, at any 

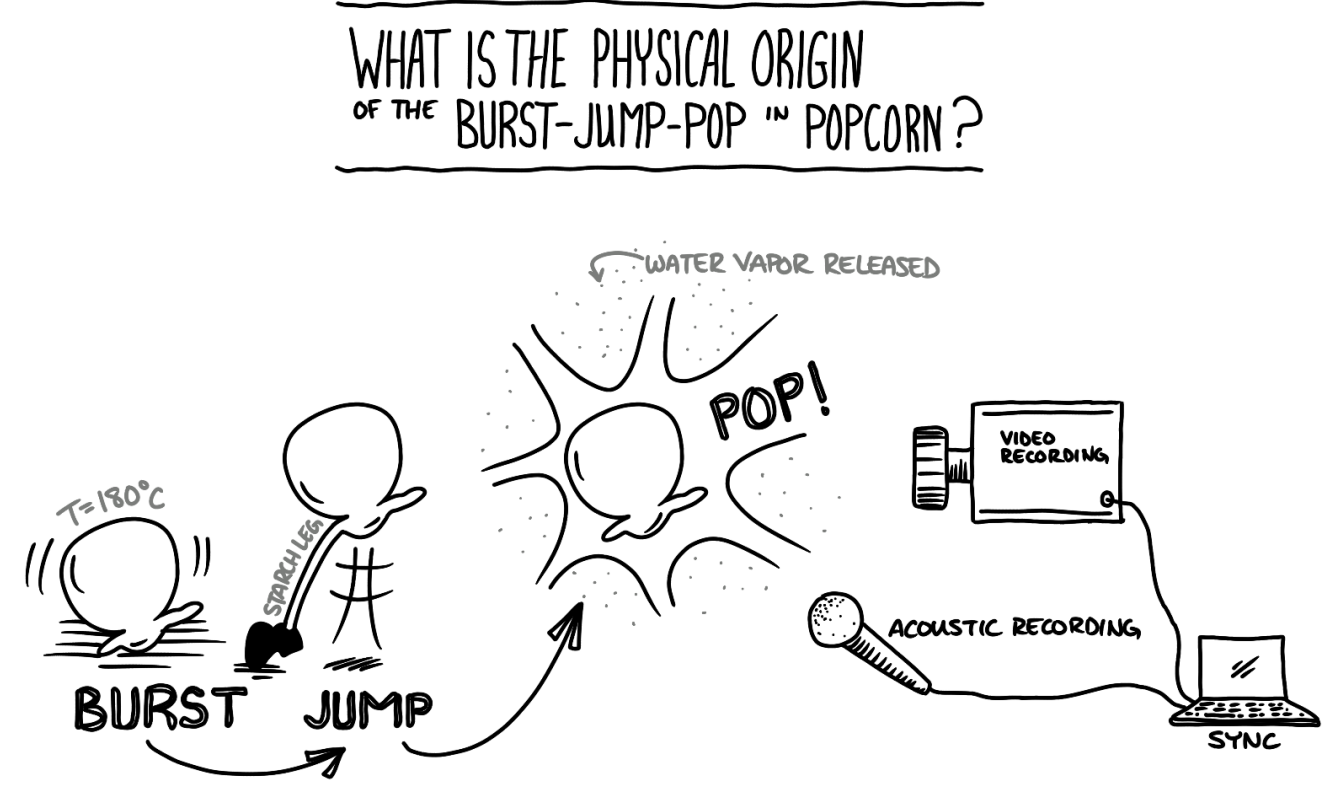

Fig. 2.2. Sketchnote of the abstract of the paper Popcorn: critical temperature, jump and sound [13].

point during the initial pass, I do not understand a term or concept, I consult texts, scholarly papers and/or research group websites so that I gain an understanding. This can be a rather time-consuming part of the workflow, especially if the subject matter is unfamiliar to me, but it is an essential step. After completing the initial pass of the paper, I have a good understanding of the research goals, results, and their importance. At this point I think about how the sketchnote will be structured. Also, I can then read all sections in the paper to obtain additional details that might be interesting to include in the sketchnote. From this point forward, we will discuss specific aspects of the sketchnote shown in Figure 2.3 and the choices made in its construction.

After reading the entire paper, there were several things that I wanted to include in the sketchnote. First, I did not know anything about the anatomy of a popcorn kernel, and this seemed like important information to include. I found information on popcorn structure from an online academic source ${ }^{2}$ and included a section on popcorn kernel anatomy. Occasionally I include information from other sources in my scientific sketchnotes, especially in situations like this where I need additional context for unfamiliar topics.

The second thing I wanted was a single sketch that illustrates the main result of the paper. Unfortunately, I was unsuccessful. There is a useful concept from advertising that can be used in sketchnotes: the hero image. In an advertisement, a hero image is one displayed prominently on the page and it is meant to attract the reader to read all the content in the ad. In the paper, if you find a figure that is a good summary representation answering the

\footnotetext{
${ }^{2}$ https://sites.psu.edu/siowfa15/2015/09/18/the-science-of-popcorn/ accessed 06.26.2020.
} 


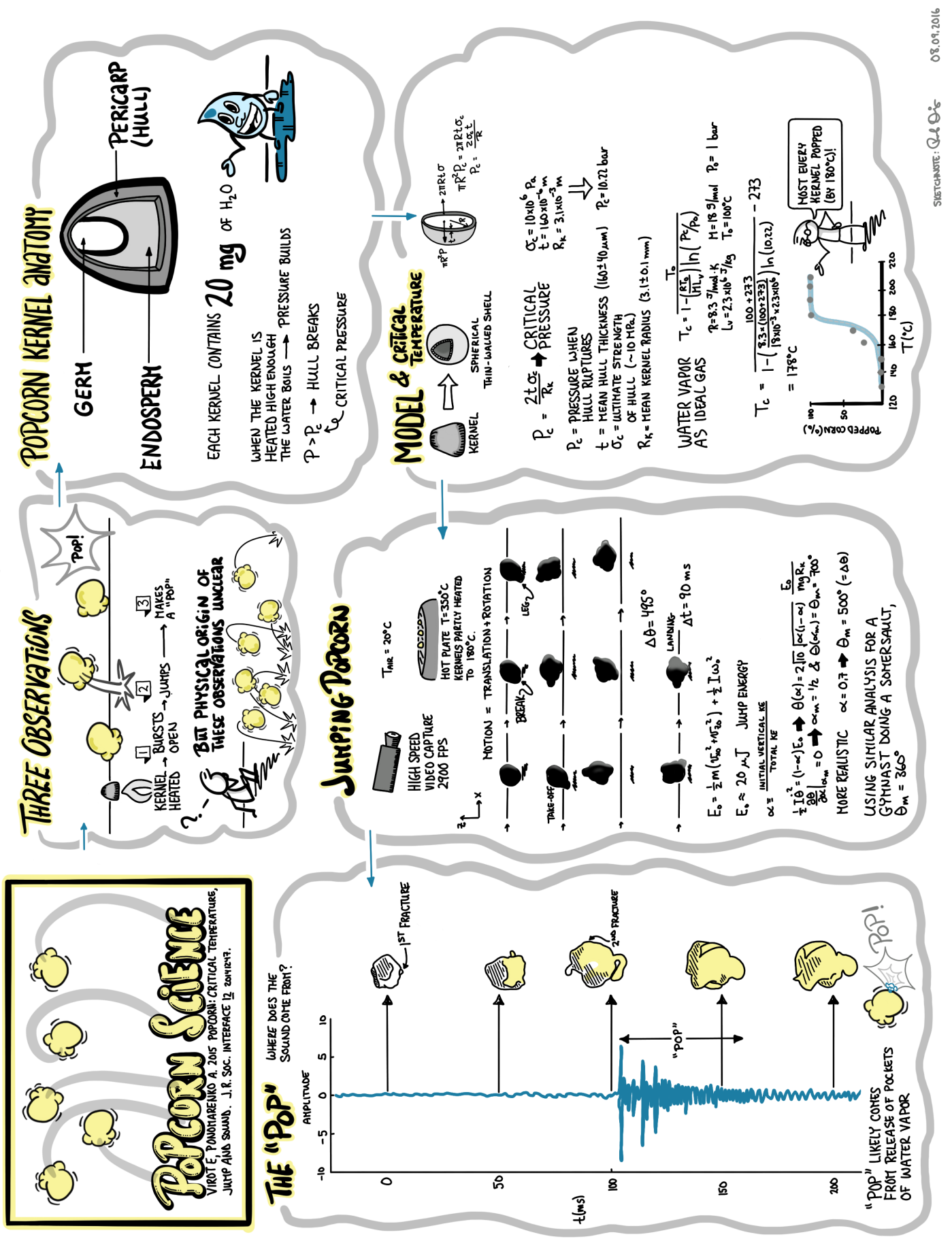

Fig. 2.3. Sketchnote of the paper Popcorn: critical temperature, jump and sound[13].

research question posed, you may consider using that as the hero image for your sketchnote. Figures 1 and 6 in the paper [13] summarize well the results, yet no single figure captures it 
all. In the final sketchnote, individual data sketches based on each figure were included. No single hero image was used. We will see examples of sketchnotes that have a hero image in the last chapter of this book (e.g., Figure 5.3). I also included elements of Figure 3 from the paper since it was a good visualization of dynamics of the jumping popcorn.

The third thing that I wanted to include in the sketchnote was some analysis. I liked the straightforward calculation of the temperature at which the kernels pop based on simple thermodynamics. I also liked the application of classical mechanics to the rotational and translational motion of the popcorn during its jump. Both are included in specific sections of the sketchnote titled "Model \& Critical Temperature" and "Jumping Popcorn", respectively. In each of those sections I present details from the paper that others might not include. There are also plenty of details that I did not include. It is purely a matter of personal preference and what interests you that influences the choices of what to include in your sketchnote.

Technical documents frequently contain data and fits of models to data. Several questions arise regarding data figures in publications. Should you include data plots in your sketchnote? How many plots should you include? Is it ok to sketch data? First, it is unnecessary to include many data plots in your sketchnote. In fact, in keeping with the sketchnote being about big ideas as opposed to a comprehensive record of the source content, you may want to limit the number of data figures in your sketchnote. I seldom have more than one or two data sketches.

When you do want to include a data sketch, it is important to remember that you are not making a document for the official scientific record. You are making notes for yourself. Sketches of data in your sketchnote are representational only. Therefore, they do not have to comply with the same rules of scientific data presentation, but only convey the main idea of what the data is saying. If you intend to share your sketchnote with others, then you can include a disclaimer such as "sketches of data in this sketchnote are representational only and should not be interpreted as a record of an actual measurement."

Data sketches need not look identical to the way they are presented in the original paper. For example, I drew a modified representation of Figure 3 from the paper [13] in the section labeled "Jumping Popcorn" so that it fit better in that section. I completely reoriented Figure 6 into the form seen in the section labeled "The "Pop" in the sketchnote. Reorienting the sketch enabled it to fit in the remaining space on the page.

The flow of information in the sketchnote follows the structure of the content in the technical paper. It starts with a title in the upper left-hand corner with cartoon jumping popcorn for visual interest. Next, a version of the visual abstract we drew in Figure 2.2 is included in the first panel and poses the research question being asked and answered in the paper. The popcorn anatomy is included in the following container and gives just enough description of this unfamiliar topic so that I can understand the paper. The measurements and data analysis are presented in the next three sections/containers as the sketchnote flow continues counter-clockwise, ending underneath the title.

Now that you've read in some detail my approach to sketchnote a technical publication, we can summarize it as follows. 


\section{Written Technical Content}

1. Read and understand the paper.

a) Read title and abstract and make a sketchnote.

b) Skim paper content ${ }^{3}$;

c) Research unfamiliar terms.

d) Complete reading the paper carefully.

2. Decide what to include in the sketchnote:

a) If topic unfamiliar, include background information from other sources?

b) Include which data sketches? How many? Use hero image?

c) How much theoretical/experimental detail to include? What do you want to remember?

d) How much analysis detail to include? What do you want to remember?

e) Choose structure and flow of sketchnote that makes it easiest for you to follow (e.g., paper structure or other?).

3. Create draft of the sketchnote (optional).

4. Create finished sketchnote.

Another sketchnote example based on a technical publication titled Statistics of Fractionalized Excitations through Threshold Spectroscopy[17] is shown in Figure 2.4. We will not go into the same level of detail as in the previous example, but rather highlight some of the main characteristics.

The content of this sketchnote was influenced by my unfamiliarity with the paper's subject. Morampudi's paper described how neutrons could be used to detect a quasiparticle called an anyon. Though I knew about some aspects of the paper, I did not know anything about anyons nor threshold spectroscopy. After reading the abstract, I researched the concept of anyons via several references, including Refs. [18], [19], and [20]. There was enough context in the paper on threshold spectroscopy that further research on that topic was unnecessary.

The sketchnote is divided into two major sections. The upper half includes the title and a brief review of anyons, with cartoon characters helping to explain them. The first character asks "what's an anyon?" The second half answers the question. The narrative in the characters' discussion is based on my understanding of statistical mechanics and the content in Refs [18], [19], and [20]. Using cartoon characters to explain anyons is unnecessary but it adds visual interest for me. This method for explaining scientific material to a broad audience using cartoon characters has been used, for example, in the "Cartoon Guide to..." series of books. See, for example, Ref. [21].

\footnotetext{
${ }^{3}$ I skim in the following order: introduction/conclusion/figures
} 
The bottom half contains a description of the proposed method to detect anyons in a quantum spin liquid. These panels contain a lot of text, equations and data sketches. These were all included because the method proposed was new to me, it involved something in my professional area of interest (neutron spectroscopy), and I wanted to remember details of the proposed experiment.

This sketchnote is visually more dense than that in Figure 2.3 and that density is offset somewhat by including some cartoon elements. This higher density results from anyons and quantum spin liquids being more abstract and challenging to understand for me than popcorn dynamics and thermodynamics. In cases where you want to remember details about more challenging topics, you may spend quite a bit of time working on the sketchnote (including researching the topic). Of course, this means that you will spend a lot of time with just one paper, but that time spent will result in a deeper understanding of the material and a meaningful visual reference that you can review anytime.

Exercise 2.2.1. Create a sketchnote based on the content in the paper Collective motion of humans in mosh and circle pits at heavy metal concerts[4]. Note that you can use the sketchnote you made of the title and abstract in Exercise 2.1.1 as a starting point.

Exercise 2.2.2. Create a sketchnote based on the content in the paper In-vivo biomagnetic characterisation of the American cockroach [10]. Note that you can use the sketchnote you made of the title and abstract in Exercise 2.1.2 as a starting point.

Exercise 2.2.3. Create a sketchnote based on the content in the paper A LEGO Watt balance: An apparatus to determine a mass based on the new SI[22] 


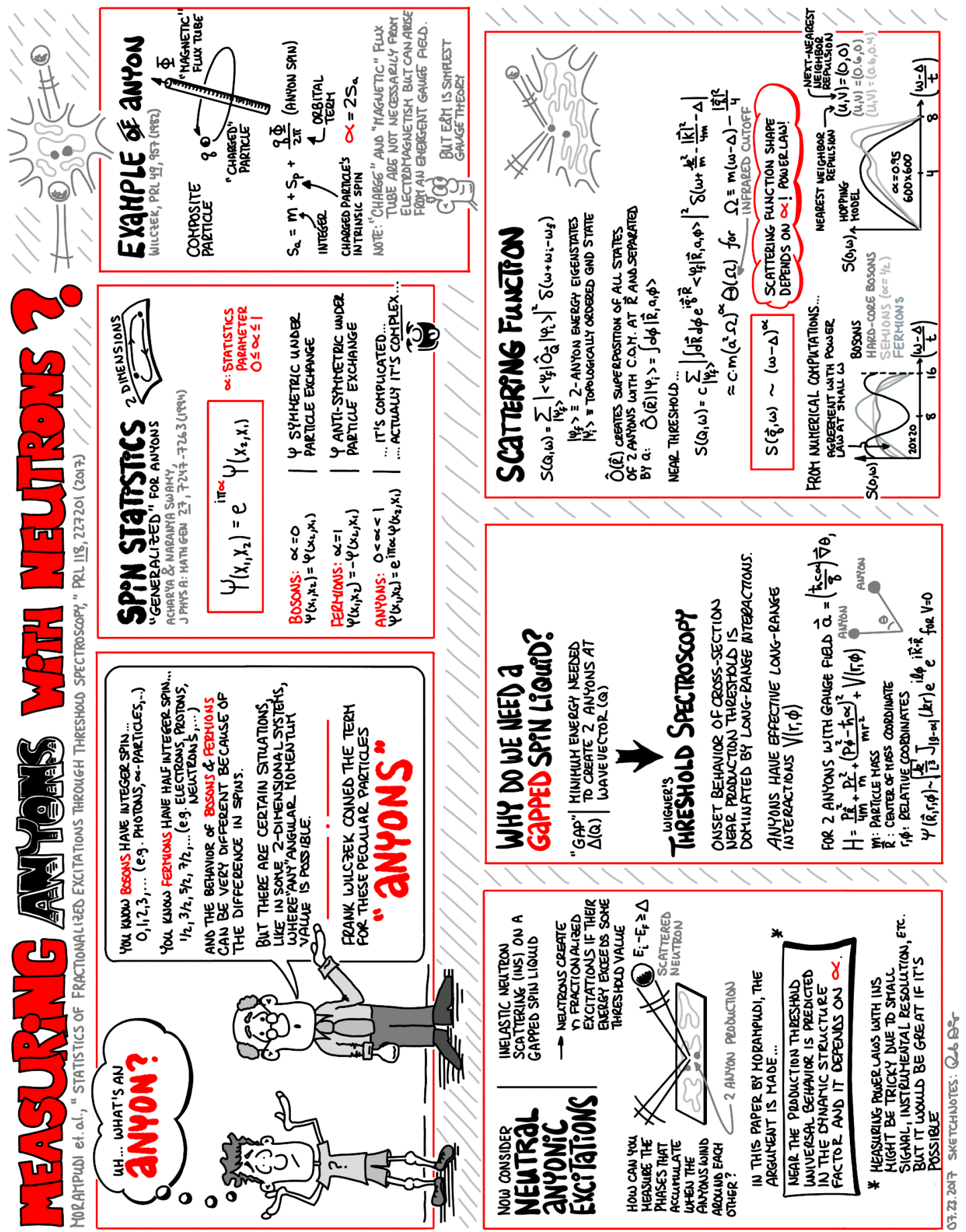

Fig. 2.4. Sketchnote of the paper Statistics of Fractionalized Excitations through Threshold Spectroscopy[17]. 


\title{
3. Technical Presentations
}

\author{
"People feel good after talks they understand." \\ - N. David Mermin, What's wrong with those talks?[23]
}

Creating a sketchnote based on a technical presentation can be one of the most difficult applications of the method and requires a workflow to be successful. This chapter describes a workflow that I developed and gives an example of its application. The result of the workflow is to obtain a good enough understanding of the talk's content that you can create a visual summary of the content.

There are three main challenges. First, if you are not familiar with the topic, then you will face the same challenges as when sketchnoting a scientific paper of an unfamiliar topic. The gap between what you know and what the speaker assumes you know could make it difficult for you to follow the presentation. Second, as opposed to sketchnoting written technical content, there is time pressure to capture the main ideas during the talk. Time pressure can lead to stress and make sketchnoting frustrating. Third, the information in the talk can be dense, both visually and verbally. When the time pressure is combined with a presentation of dense information, the result can be a harrowing sketchnoting experience. The speaker may talk at too rapid a pace or display data-heavy slides too briefly, making it difficult to distinguish the big ideas from less important details. Unfortunately, this is an error that many speakers make when creating a technical presentation. It can be attractive for a speaker to include as much information on their research effort as possible rather than to tell a compelling story with a beginning (importance of question), middle (approach to answering question), and end (results and implications of the answer). Moreover, speakers often assume a common background and expertise, thus leaving out fundamental information that you require for understanding even if you have some familiarity with the topic. A talk often is only self-contained for the speaker who created it. You approach it with your (different) background and expertise. Therefore, preparation is the key step in the following workflow:

1. Preparation: Read and understand talk title and abstract.

2. During the talk: Create a rough sketchnote.

3. After the talk: Create a final sketchnote. 


\section{Technical Presentations}

In the best circumstances, when you know the topic well and the speaker presents the talk in a deliberate, well-paced and easy-to-follow manner, you may be able to create the final sketchnote during the talk. Preparation may be unnecessary. It is also possible that you may be able to create a final sketchnote during the talk after you have done adequate preparation. I have experienced both situations. However, in most cases I do all three steps: preparation, rough sketchnote and final sketchnote. In this chapter, you will learn the complete workflow and see its application to a technical presentation you can view on-line.

\subsection{Preparation}

A major challenge when sketchnoting a technical talk is to recognize what is important and what is of lesser importance. This is especially difficult if the topic is unfamiliar to you. In that case, everything seems important. Becoming familiar with essential concepts before the talk helps in a "live" sketchnoting situation.

Preparation may require nearly no time to several hours, depending on how familiar you are with the topic. The benefit of preparation obviously goes beyond sketchnoting. When you go to a talk prepared, you can be more engaged with the talk and get more out of it. Regardless of your familiarity, the first step is to read and understand the talk title and abstract. You can make a quick sketchnote based on these as part of the preparation, as discussed in the previous chapter. If there are unfamiliar terms or concepts, then you can consult texts, scholarly papers and/or research group websites to gain some understanding. Reviewing the speaker's publication list, especially published work closely related to the talk, can be very helpful. You can become familiar with recent results, experimental techniques, analysis methods, and other researchers doing related work (from the references in the publications) by skimming or reading selected publications authored by the speaker. Often the speaker's research group's website will include links to recent presentation slides. Previous presentations can be a good resource as you prepare.

While preparing and doing research for the talk, think about what visual vocabulary elements you might want to use in your sketchnote. A visual vocabulary is as important as a verbal vocabulary for a sketchnoter, especially in a "live" sketchnoting situation. This is true for sketchnoting technical and non-technical talks. Just as you usually do not think deeply about what word comes next when speaking to someone or when writing it on the page, you do not want to think too hard about how to sketch something. ${ }^{1}$ When sketchnoting, you need to focus on the content of the talk, rather than how to sketch a concept. A good visual vocabulary enables you to keep your focus on the content of the presentation rather than think about how to represent a concept in a quick sketch. You can prepare by brainstorming different ways to sketch concepts and terms related to the presentation title and abstract.

\footnotetext{
${ }^{1}$ In particular, when you write the word "pencil", you do not have to think about how to draw each letter in the word. However, if you draw a pencil for the first time, you have to think about how to assemble a series of lines so that it is a visual representation of a pencil.
} 
The last step in preparation is to create the title on the page with the speaker's information, date and any other information you wish to include. You may prefer to make the title in advance to save time.

\subsection{During the talk}

When the talk begins, listen for the main point. This often is part of the speaker's introductory material early on. Since you already know the title and abstract for the talk, the speaker's main point should not be a surprise to you. However, you may learn additional helpful context in the introduction. This is a good opportunity for you to sketch your interpretation of the main point or the research question being asked, which can be informed by the sketchnote you made of the title and abstract.

The speaker may also describe the structure of the talk. This can be particularly helpful so that you think about how to organize the page based on that structure. You should make note of that structure so that you can use it as you develop the sketchnote over the course of the talk.

As the talk unfolds, listen for important information that supports the main point. In a scientific talk, the speaker may present theoretical background, mathematical development of an existing or proposed theory, experimental consequences of theory, description of experimental techniques used in the research, results, conclusions and new areas to be explored. Visual information that you can consider sketching include drawings of apparatus, diagrams that explain concepts, plots of theoretical models, data and/or model fits to the data. You have to determine which of these to include in your sketchnote based on the relative importance to the main point. This is your personal choice, but often you need to decide quickly. If the pace of the talk is rapid, then it may be challenging to make these sketches in real-time. It is helpful if you developed some visual vocabulary so that you can sketch concepts quickly. However, even the best preparation and most complete visual vocabulary will not help if the slides are presented too briefly. In some situations, I use the camera on my cell phone to take snapshots of some slides during the talk. ${ }^{2}$

During the talk, it is likely that there will be topics discussed or examples given that are either unfamiliar or discussed too quickly, in spite of your preparation. You have a choice to include these. If you do decide that they are important enough to include, then you will need to do some research after the talk.

\subsection{After the talk}

The last task is to create the final sketchnote. This may require resolving concepts that were unfamiliar or unclear during the presentation. There are two things you can do. First, right after the talk concludes, you may have an opportunity to ask the speaker questions.

\footnotetext{
${ }^{2}$ Certain venues prohibit photography of any kind. Get permission before taking photos of slides. Also, with the increasing number of talks being offered live in a virtual manner, you can take screenshots of slides as long as the speaker has given permission.
} 


\section{Technical Presentations}

Take advantage of this opportunity. You can also show the speaker your sketchnote after the Q\&A session ends with the audience and ask specific questions for clarification. In my experience, speakers are often excited to see the novelty of a sketchnote of their presentation. It shows the speaker that you were paying attention to their talk and are deeply interested. If the speaker presented an unfamiliar topic or example, this is an excellent opportunity to find additional references so that you can include information from those in your final sketchnote. Feel free to request the speaker's email address and offer to send him/her a photo of your final sketchnote, if he/she is interested. That connection also allows you the opportunity to follow up with additional questions for clarification via email. Second, you can educate yourself on unfamiliar topics by following up on relevant references cited during the talk. Information from other sources, perhaps read during your preparation phase, can also provide needed background for your final sketchnote. You may decide that there is important background that was not provided during the presentation but is needed for you to have as an enduring reference in your sketchnote. Recall the example from the previous chapter in Figure 2.4 based on Ref. [17]. In that example, half of the sketchnote was background that was not included in the paper being sketchnoted. Remember that the sketchnote you make is a reference for you. What you choose to include is up to you.

The next step is to review your rough sketchnote and determine which sketches to include in the final version. In particular, if there is one figure - either an illustration of a major theme, concept or data that summarizes a major result - you can consider using that as a hero image as described in the previous chapter. If you choose to include a hero image, you will need to consider how big to make it and where to place it. The choices you make regarding how much theoretical and/or experimental detail to include, how much analysis detail, and the conclusions are based on what you want to remember. You need not include everything from your rough sketchnote in the final version.

The next step is to choose how to organize the content. If you are satisfied with the organization of the content from your rough sketchnote then you can simply use that. However, you may decide afterwards that a different organization of the content will communicate the information more effectively for you.

Once you have determined the content to include in your final sketchnote and the organization of the content, you can draw the final version. This is, perhaps, the easiest part of the workflow for me. It entails carefully sketching things again so that they are neat and easy to understand, and writing things so that they are legible. For written words, I emphasize major points and annotate containers and groupings of ideas with carefully drawn large, bold text to draw attention to those elements.

The steps in the workflow for sketchnoting a technical talk described above are listed below.

1. Preparation: Read and understand talk title and abstract.

a) Read the title and abstract.

b) Research unfamiliar terms and concepts. 
c) Scan speaker's publication list, particularly work closely related to that described in the title and abstract.

i. Read recent related papers by the speaker.

ii. Review recent related presentation slides by the speaker.

d) Develop a visual vocabulary for unfamiliar terms.

e) Create a sketchnote of the talk title and abstract.

f) Start your rough sketchnote of the talk by making the title with the speaker's information and date.

2. During the talk: Create rough a sketchnote.

a) During the introductory part of the talk, listen for the main point. What is the overarching question and why is it important? Sketch your interpretation.

b) In the introductory part of the talk, listen for the structure of the talk. Write down the structure as an outline.

c) For slides with data important to the overall message, make a data sketch. If the speaker is moving too quickly, take a photo of the slide. ${ }^{3}$

d) Make simple sketches of concepts, experimental techniques, apparatus, data and fits, when they seem important. ${ }^{4}$

3. After the talk: Create final sketchnote.

a) If you have questions not answered during the Q\&A session (or there is no Q\&A session afterwards), ask the speaker your questions for clarification.

b) If a topic is unfamiliar, consider including background information from other sources.

c) Determine which data sketches to include. How many? Use a hero image?

d) Determine how much theoretical/experimental detail to include. What do you want to remember?

e) Determine analysis detail to include. What do you want to remember?

f) Choose the structure and flow of the sketchnote that makes it easiest for you to follow (e.g., paper structure or other?).

\footnotetext{
${ }^{3}$ Note: Photography may not be permitted in certain situations. You should ask beforehand.

${ }^{4} \mathrm{~A}$ speaker may argue that everything in the talk is important but remember that your goal is to extract the most important aspects of the talk for you. Therefore, not all information presented should be recorded in your sketchnote.
} 


\section{Technical Presentations}

\subsection{Example: Sketchnoting a live talk}

We will demonstrate this workflow by applying it to a "live" talk. We will create a sketchnote of a scientific talk that was recorded and is available for you to view. The title of the talk is Ultracold Molecules: From Quantum Chemistry to Quantum Computing by Dr. Alan Jamison and it is about 1 hour long.[24] Dr. Jamison presented this talk to the Perimeter Institute at the University of Waterloo on May 6,2020. You can view the video by clicking here. ${ }^{5}$ The abstract for his talk is listed below.

Cooling atomic gases to ultracold temperatures revolutionized the field of atomic physics, connecting with and impacting many other areas in physics. Advances in producing ultracold molecules suggest similarly dramatic discoveries are on the horizon. First, I will review the physics of ultracold molecules, including our work bringing a new class of molecules to nanokelvin temperatures. Chemistry at these temperatures has a very different character than at room temperature. One striking effect is our recent result using spin states of reactants to control chemical reaction pathways.

Our first step is to read the title and abstract. A simple sketchnote representing this is shown in Figure 3.1. Drawing a quick sketchnote of the abstract and title is a useful exercise because it forces you to start to think about how to visualize the paper's concepts and highlights the main points at the outset. The description in this abstract is a highlevel summary. Since I did not know much about ultracold molecules, I did a quick web search and learned that there are a couple ways to achieve ultracold molecules. They can be made (1) by cooling a gas of stable molecules, and (2) they can be made by combining ultracold atoms to form molecules. ${ }^{6}$ The resulting sketchnote shows a simple sketch of two cold atoms and a cold molecule, the title and a couple statements about quantum chemistry interpreted from the abstract.

Since this was an unfamiliar topic, I read several online resources and I skimmed a related publication by the speaker.[25] It provided exposure to terminology and concepts in the field that could be important in understanding the talk better. In particular, the concept of sympathetic cooling described in the paper provided needed context as a demonstration of what one can do with ultracold molecules.

I did not have to spend much time thinking about a visual vocabulary after skimming online resources and a paper. The main images consisted of simple molecular models (ball and stick models), potential/energy-level diagrams, and straightforward data plots. I sketched these many times before during technical presentations.

The final step in preparing for sketchnoting the talk is to make the title for the sketchnote, shown as the bold handwriting in the upper left-hand corner of the page in Figure 3.2. This is optional but I frequently do this step. It forces me to think subsequently about content

\footnotetext{
${ }^{5}$ https://www.perimeterinstitute.ca/videos/ultracold-molecules-quantum-chemistry-quantum-computing accessed June 28, 2020.

${ }^{6}$ https://jila.colorado.edu/yelabs/research/ultracold-molecules (accessed June 28, 2020).
} 

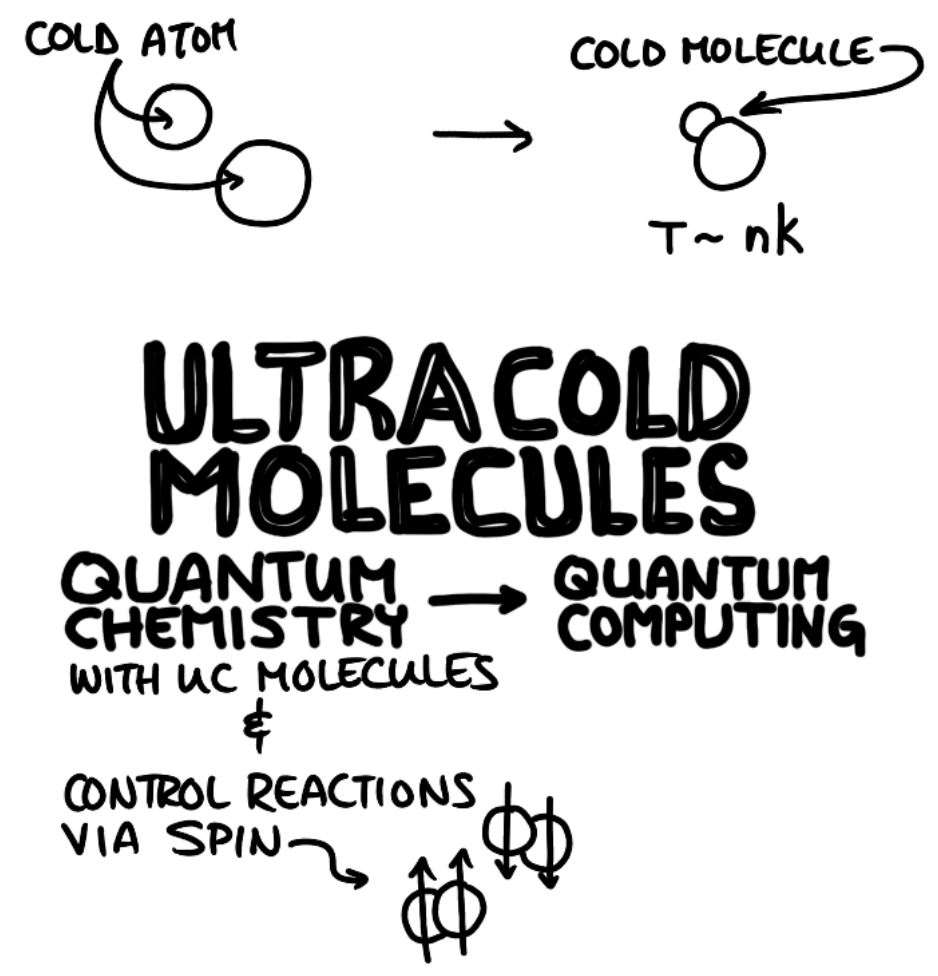

Fig. 3.1. Sketchnote of the title and abstract from Ref. [25].

layout and how to arrange information on the page based on how I've drawn/written the title.

During the talk I found the material was presented at a reasonable level and at a comfortable pace for me. There was only one example given that required further research. In that case (spin-controlled chemistry/hydrolysis), I made note of the Ref. [26] provided on the slide. Part of the issue was that I could not resolve several features in the figure presented on the slide in that example.

The time that the speaker spent on each slide was long enough to allow me to make rough sketches of important diagrams and data during the talk. For many technical presentations I have attended, this has not always been the case. In those cases, I used the camera on my cell phone to take snapshots of some slides during the talk. ${ }^{7}$

My rough sketchnote is shown in Figure 3.2. In order to distinguish this from the final sketchnote, I used the digital equivalent of a pencil to emphasize that these are rough notes. This messy page of notes is a typical example of how my rough notes appear when taken during a presentation.

After the talk finished, I reviewed my rough sketchnote. As mentioned above, there was only one example that was unclear to me and I made note of the reference. I reviewed the

\footnotetext{
${ }^{7}$ I only do this after asking if it is acceptable to do so.
} 


\section{Technical Presentations}

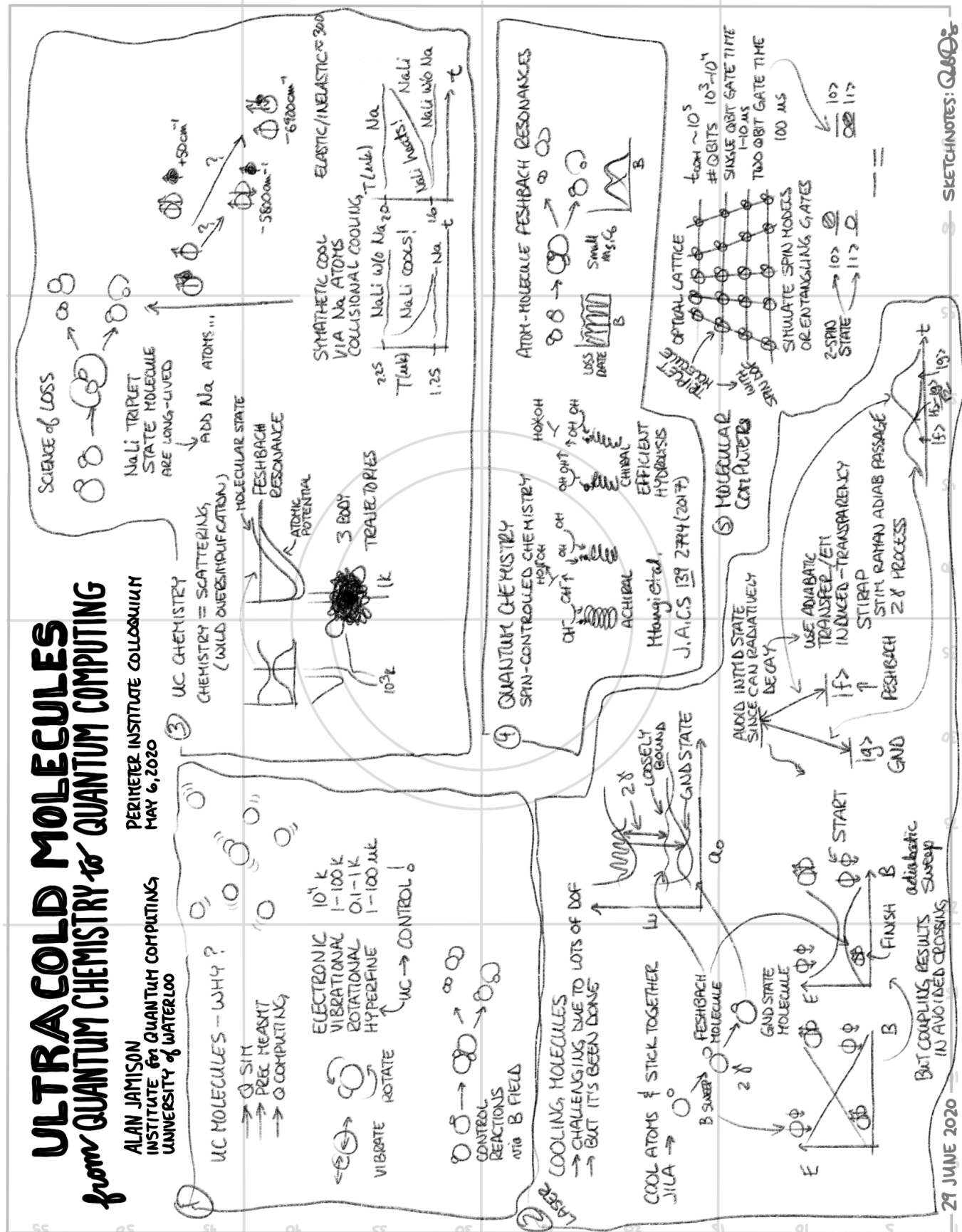

Fig. 3.2. Rough digital sketchnote based on a colloquium by Dr. Alan Jamison.[25]

reference afterwards ([26]) and found the figure that was not clear in the speaker's slide. In fact, I found that example so interesting (i.e., an application of chiral materials) that I added more detail in the final sketchnote. A marvelous flexibility of this note-taking method is that, since the notes are for you alone, you are free to let your intellectual interests take you 
in different directions and add any related items that pique your curiosity.

As I reviewed the rough sketchnote, I looked for figures that captured the message. This, like the other choices you make when sketchnoting, is a personal choice. You should ask yourself "is this something that I want to remember?" I made quite a few sketches in the section describing the process used to make ultracold molecules. The size of this section and the number of sketches reflects the amount of time that the speaker spent describing that process in detail. However, on further review I decided that it was unnecessary to include all that detail. Note that this is a common occurrence in my rough and finished sketchnotes from technical talks. I often have more detail in the rough notes compared with the final sketchnote. I use the time after the talk to review my rough sketchnote and discern what should be included in the final sketchnote. Once I decide which sketches to include, I sketch them more carefully so that they are easy for me to understand with a quick view.

The final major design choice that I made was to structure the information flow in two columns and group major themes in numbered, rectangular containers. I then wrote the text more carefully so that it was more legible and I added emphasis in several locations. The words that describe the topic in each container were drawn neatly in bold. This draws attention to the content in the individual container and distinguishes it from the supporting text within. I also chose to draw some words in cartoon form. In particular, see the writing in container number 2: "Instead...first cool the atoms then put them together!" This borrows a trick from comics to emphasize bold statements or concepts. The notion of first cooling atoms and then assembling them was new to me. The conventional way of starting with molecules and cooling them to low temperatures is an obvious approach. But the speaker did an excellent job explaining why it is so difficult to cool molecules to $\mu \mathrm{K}$ temperatures. To highlight this novel way to create ultracold molecules, I used block letters and added drop shadows to them in that phrase.

You have learned the workflow and seen a detailed example. Now it is your turn to put the workflow into practice. You can choose to apply the workflow to a technical talk that you attend or practice with any of the numerous technical talks available on-line.

Exercise 3.4.1. Create a sketchnote based on the talk by Itai Cohen titled From Flies, to Mosh Pits and Mecca: Predicting the Collective Behavior of Organisms from June 20, 2016. Note that your work from Exercise 2.2.1 will be helpful to review before watching this presentation. ${ }^{8}$

Exercise 3.4.2. Create a sketchnote based on a technical presentation. You can sketchnote a "live" talk or choose one from video libraries available on-line, such as from the Perimeter Institute (physics) $)^{9}$, UC Berkeley (physics) ${ }^{10}$, or Stanford (biology) ${ }^{11}$.

\footnotetext{
${ }^{8}$ https://youtu.be/s4InWqgsxCc accessed July 5, 2020

${ }^{9} \mathrm{https} / / / \mathrm{www}$.perimeterinstitute.ca/video-library accessed July 10, 2020.

${ }^{10} \mathrm{https} / / /$ physics.berkeley.edu/resources/colloquia-and-videos accessed July10, 2020.

${ }^{11}$ https://biox.stanford.edu/videos accessed July 10, 2020.
} 


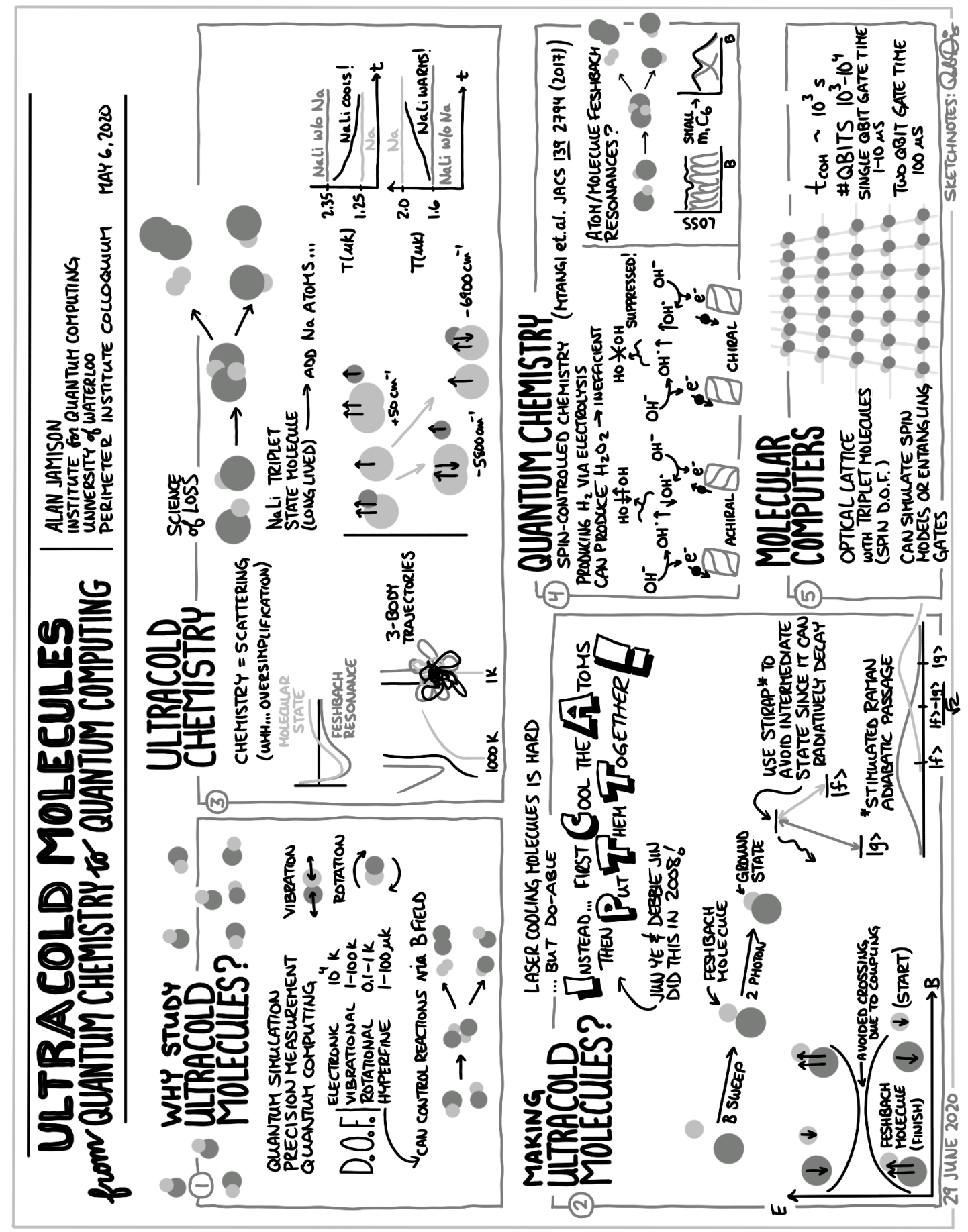

Fig. 3.3. Final digital sketchnote based on a colloquium by Dr. Alan Jamison.[25] 


\section{Presentation Planning \& Visuals}

"The longer you give your audience something interesting to look at, the longer they will give you their mind."

- Dan Roam, Draw to Win[27]

You have seen how sketchnotes can be used to make a visual recording of technical content. Two other applications of sketchnotes are planning and presentation.

\subsection{Planning tool}

You can use a sketchnote as a planning tool. For example, if you are writing a paper or a report, you can begin by planning its organization and how to present the ideas in a sketchnote. Similarly, you can plan the content and organization of a presentation using a sketchnote. This additional step in the workflow essentially replaces the standard approach of starting with an outline. In fact, this is a type of "visual outline." I find that sketching my ideas first on a blank sheet of paper before typing the first word in a report or creating the first slide promotes a different way of thinking that results in a more effective process of content creation. When you do your visual brainstorming on a digital platform or using index cards, it is easy to rearrange your ideas quickly. ${ }^{1}$

As an example of a planning sketchnote, I created the sketchnote in Figure 4.1 as a first step to plan the content of an article that I wrote about sketchnotes.[28] I drew this sketchnote to capture my thoughts on what to include as well as how to organize it. I did not start typing the manuscript before I made the sketchnote. This can be a valuable time-saving step.

\subsection{Presentation visual}

The other application - and the one that we focus on in this chapter - is as the main visual in a presentation, rather than slides in a slide deck. This extends your planning described above from brainstorming on the page to creation of the final visual. In this case, the sketchnote must be prepared carefully so that it can be understood by everyone in your audience. The writing must be legible and the drawings must be clearly identifiable. But first, why would you want to make a sketchnote presentation visual?

\footnotetext{
${ }^{1}$ In fact, I created a very short sketchnote summarizing the overall structure of the book, and several additional detailed sketchnotes for individual chapters.
} 
4 Presentation Planning \& Visuals

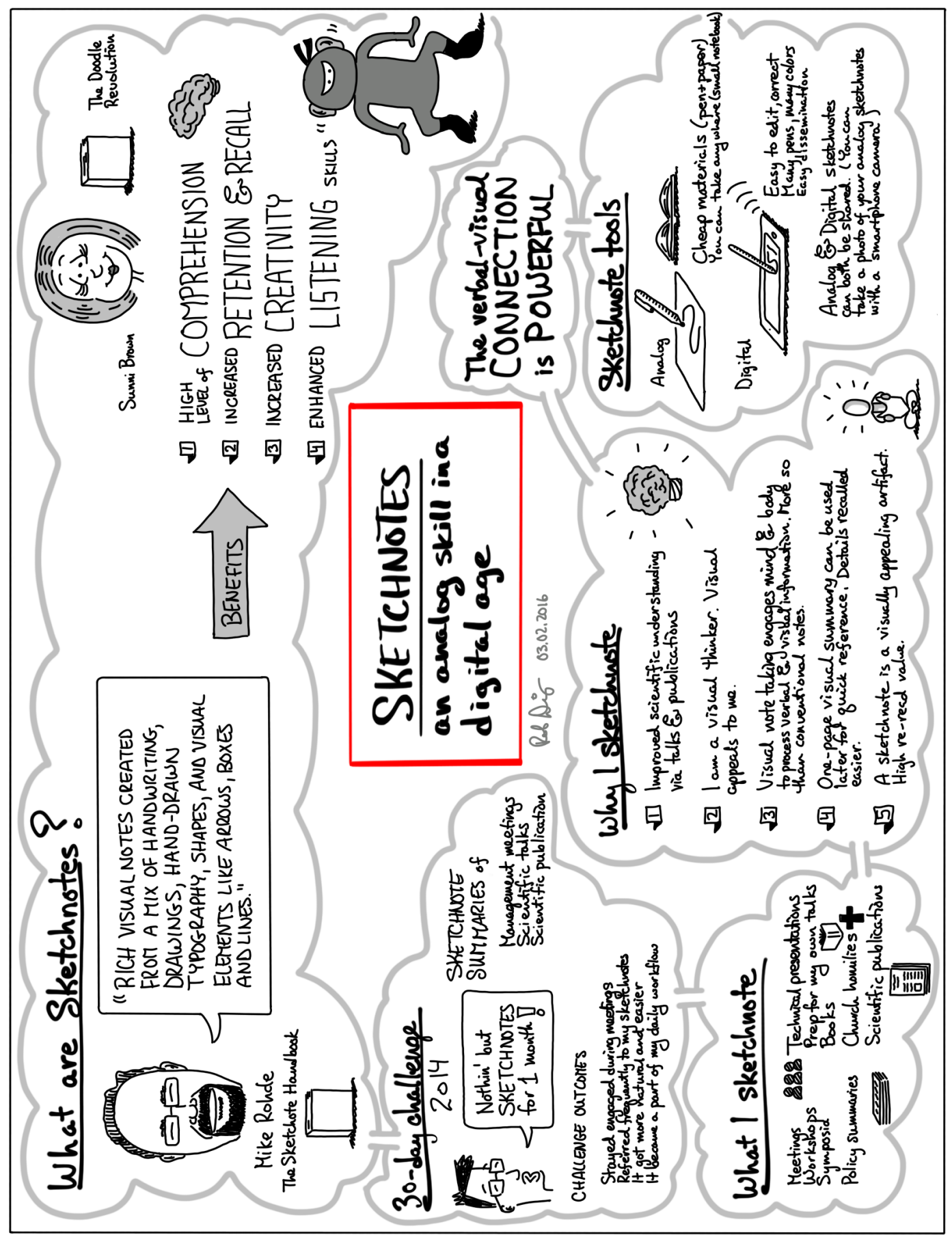

Fig. 4.1. Sketchnote used to plan a published article.[28]

There are several reasons why you should consider using a sketchnote as your presentation visual. First, it shows your audience that you care that they understand and remember 
your message when you bring paper copies of your sketchnote. Alternatively (or in addition), you can display a QR code in your presentation that links to an on-line electronic version. When you project the QR code at the end of your presentation, the audience can scan the code with their smart phones from their seats and download the visual instantly. Second, sketchnotes are made of hand-drawn elements and it has been suggested that handdrawn visuals are more engaging and accessible. In Dan Roam's popular book Show and Tell: How Everybody Can Make Extraordinary Presentations[29], he cites several advantages. The pros of drawing, according to Roam, are "With practice, these require very little time to create; Show exactly what we want; Are "warm" and inviting to look at; Show a human touch; Easy to keep simple."[29, p.217] He cites the following cons: "Require basic drawing skills; Can become overly "cute"-which is mostly a problem in reports and pitches." My experience incorporating hand-drawn visuals and the reactions of audience members supports most of Roam's points. In particular, the feedback from audience members on using hand-drawn visuals has been positive. I have not received any disparaging comments nor comments that any presentations were overly cute, even when adding cartoon characters to my presentation visuals. Nevertheless, it is not always appropriate to use hand-drawn visuals. There are some things you should not present as a sketch in a presentation, such as experimental data and model fits. If you wish to present a sketchnote with measurements, import the computer generated plots. This is straightforward with many drawing apps for tablets.

A sketchnote that describes the benefits of a presentation sketchnote visual and how to design and deliver such a presentation is shown in Figure 4.2. We discussed some of the elements presented there already so we discuss design and delivery next.

Several factors should be considered when designing your presentation sketchnote. First, a digital sketchnote (or digtal photo of your sketchnote) should have sufficient resolution so that it is easy to read when zooming into different areas. I frequently choose a digital document that is 2200 pixels $\times 1700$ pixels. This resolution not only allows you to zoom in to smaller regions in your visual, but you can print the sketchnote on paper with good fidelity. Second, you should write/draw the title of your presentation prominently and legibly. You want your audience to notice it first when they first look at your sketchnote. Third, select a layout for your sketchnote with which you are comfortable transitioning from one area to another. The randomly arranged popcorn[1, pp.113-115] layout is not a good choice in this case because it can be difficult to follow. It is best to organize information in an easy-to-follow path. A sketchnote presented to an audience for review during a talk should be easy to follow and coincide with the flow of the talk. Fourth, use as many visuals as you need to make your point, including figures, images, and hand-drawn pictures. Also, since the words you speak are the focus of the presentation content, only put enough words in your sketchnote to convey the main ideas. In summary, use less words, more visuals, and good organization.

You can present your sketchnote visual using any photo display program in which you can zoom into a region and pan to the next. If your sketchnote is hand-drawn on paper, you can take a photo or make a scan and use the digital image. Alternatively you can 


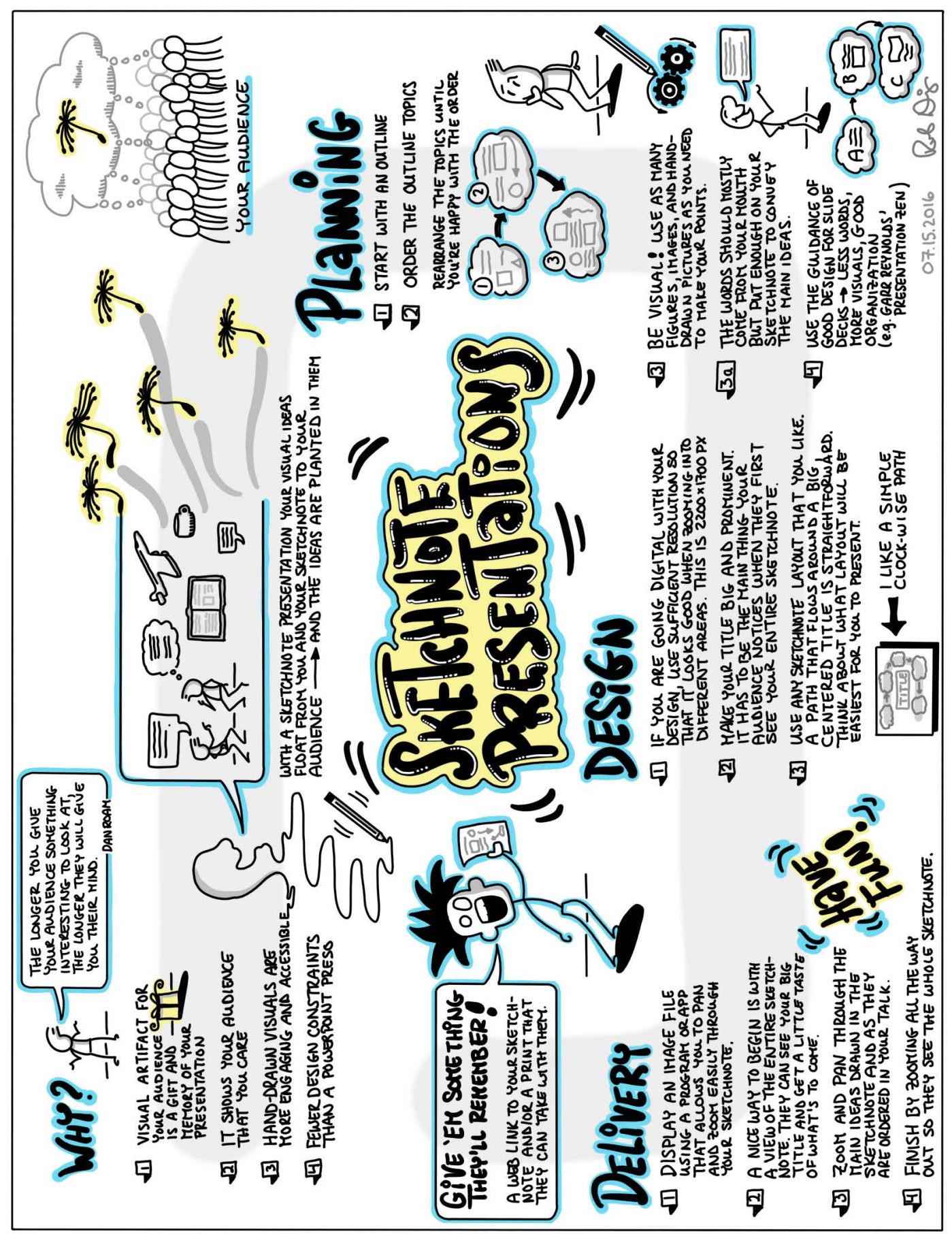

Fig. 4.2. Sketchnote summary of how to design and deliver a presentation using a sketchnote as the primary visual.

create a digital sketchnote on a tablet. I have used an application called Tawe $\mathrm{T}^{2}$ to present

$\overline{{ }^{2} \text { http://www.tawe.co/ accessed July 3, } 2020 .}$ 
my sketchnote. ${ }^{3}$ This application allows you to plan your path through the sketchnote in advance and control movement about the image.

When presenting your sketchnote, you can begin with a view of the whole sketchnote and then zoom in for a close-up view of the title. That gives your audience a hint of what's to come during your presentation. You zoom and pan through the main ideas drawn in the sketchnote as they are ordered in your talk. You can finish your talk zooming all the way out so that your audience can see the entire sketchnote. This is is a particularly good way to finish your talk because the audience is reminded visually of everything you just spoke about. For the Q\&A session afterwards, you can simply zoom into any relevant region as you provide a verbal response. The advantage over slides is that you need not flip through numerous slides in your slide deck to find the right one.

An example of a sketchnote used as the primary presentation visual is shown in Figure 4.3. Since this talk discussed the importance of innovation in instrumentation design, it included more technical drawings than plots of data. There was one quantitative plot. In that case (upper right hand corner of the sketchnote), I bent the rule by including a sketch of the data. In fact, I imported the figure into the digital sketchnote and traced the figure in a style that matched the rest of the sketchnote. A reference to the source of the figure is included below the sketch. The content for this sketchnote is organized in a modular layout. The flow of the talk began with the title in the center and moved clockwise from the upper left hand panel, finishing in the lower left hand panel. In each of the four panels, I zoomed into several areas during the presentation to highlight specific details.

Figure 4.4 shows another example of a sketchnote visual I used in a presentation to a community of technical experts to describe the development of a neutron scattering instrument at NIST. Though it was largely a historical talk, I included significant technical detail, including several quantitative figures. I created the sketchnote digitally and imported data plots, schematics, and a photograph. The flow of the sketchnote follows a counter-clockwise direction, with small arrows indicating the path.

\footnotetext{
${ }^{3}$ Any mention of commercial products is for information only; it does not imply recommendation or endorsement by NIST.
} 


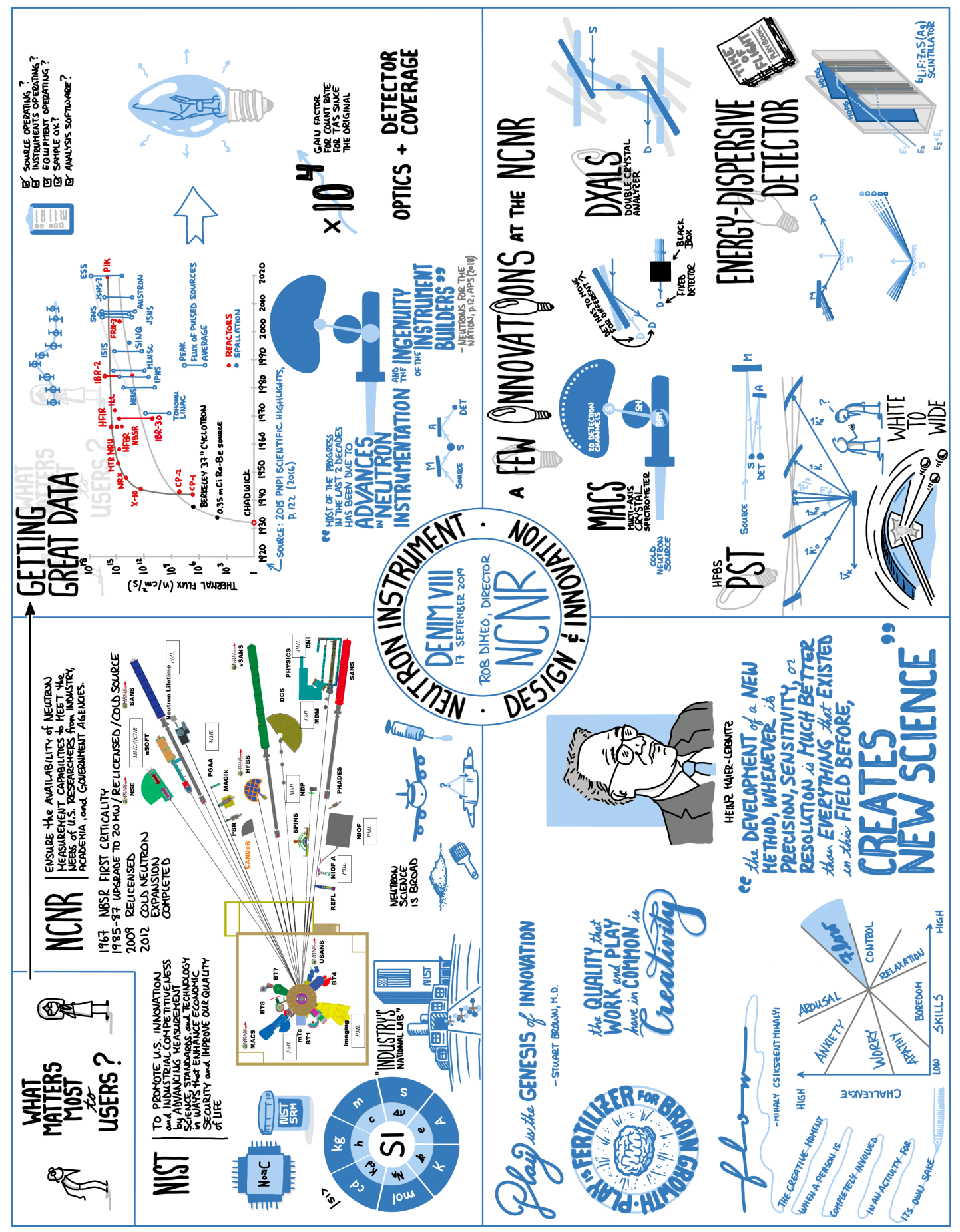

Fig. 4.3. Sketchnote used as the primary visual in a presentation at the 2019 DENIM conference.[30] 


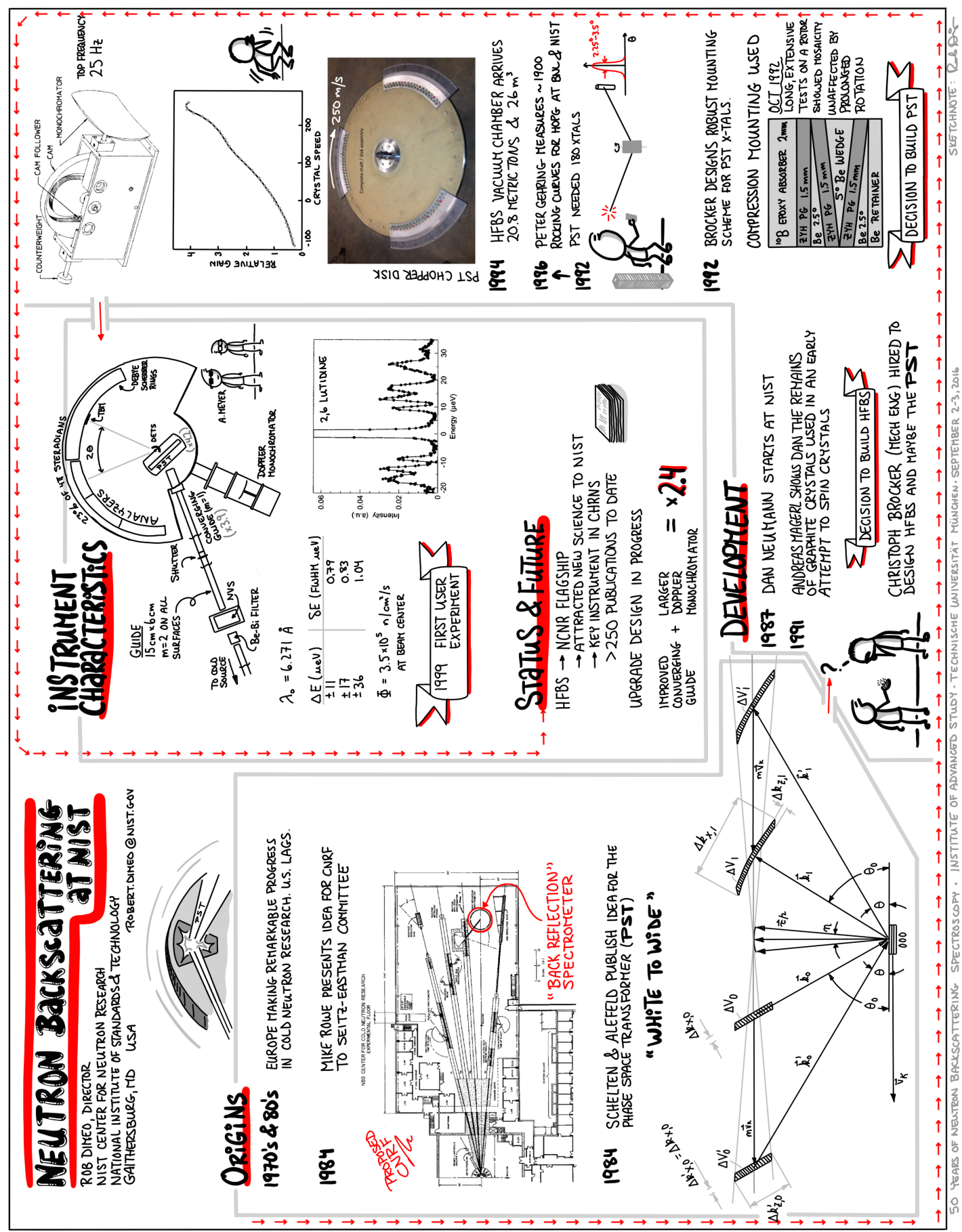

Fig. 4.4. Sketchnote used as the primary visual in a presentation at the 2016 conference in Garching titled 50 years of Neutron Backscattering Spectroscopy.[31] 



\section{Selected Science Sketchnotes}

In this final chapter, I share a selection of science sketchnotes I have created over the years. Many of these are based on publications but several are based on technical talks. The purpose of presenting these sketchnotes is two-fold. For the publication-based sketchnotes, you can see how I chose to attack the general problem of representing the content in a way that I would remember and want to review. Also, you can see the progression of my technique starting with my first sketchnote of a technical talk in Figure 5.1. You can find all of my public science sketchnotes at the following URL: https://flic.kr/s/aHskAffbgK. 

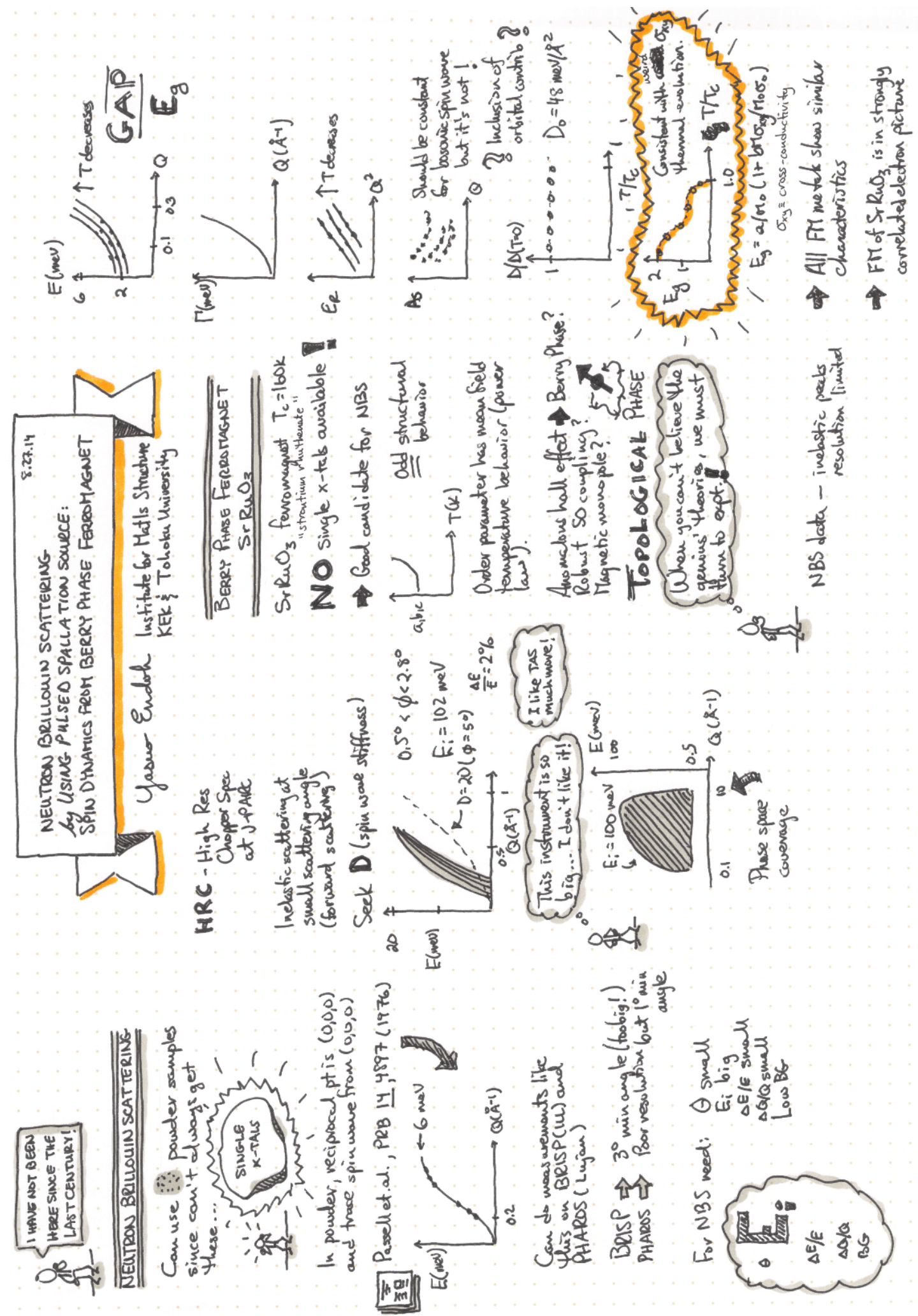

Fig. 5.1. Sketchnote made with pen on paper based on a scientific seminar given by Yasuo Endoh in 2014 at the NIST Center for Neutron Research. 


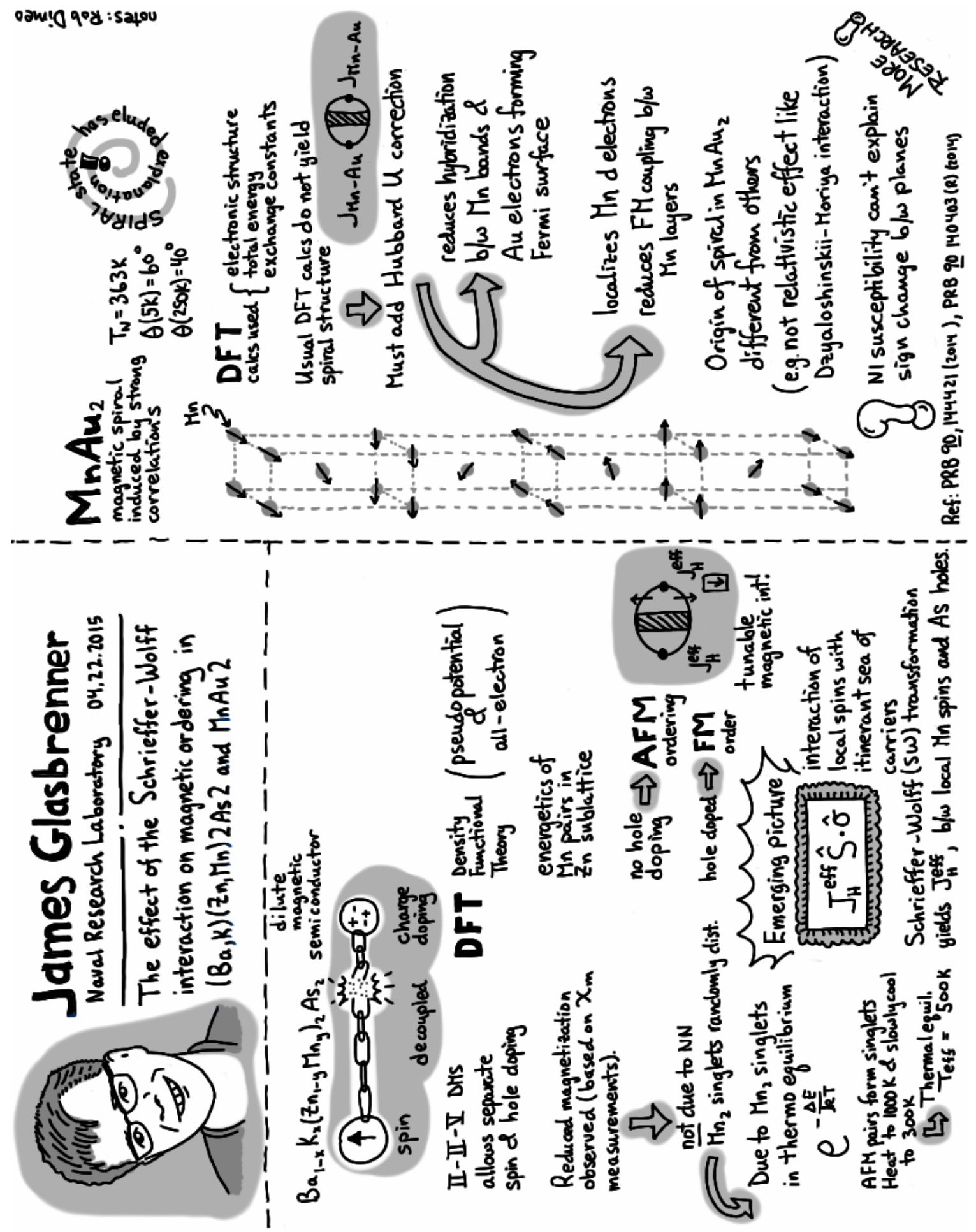

Fig. 5.2. Digital sketchnote made using a tablet based on a scientific seminar given by James Glasbrenner in 2015 at the NIST Center for Neutron Research. 
5 Selected Science Sketchnotes

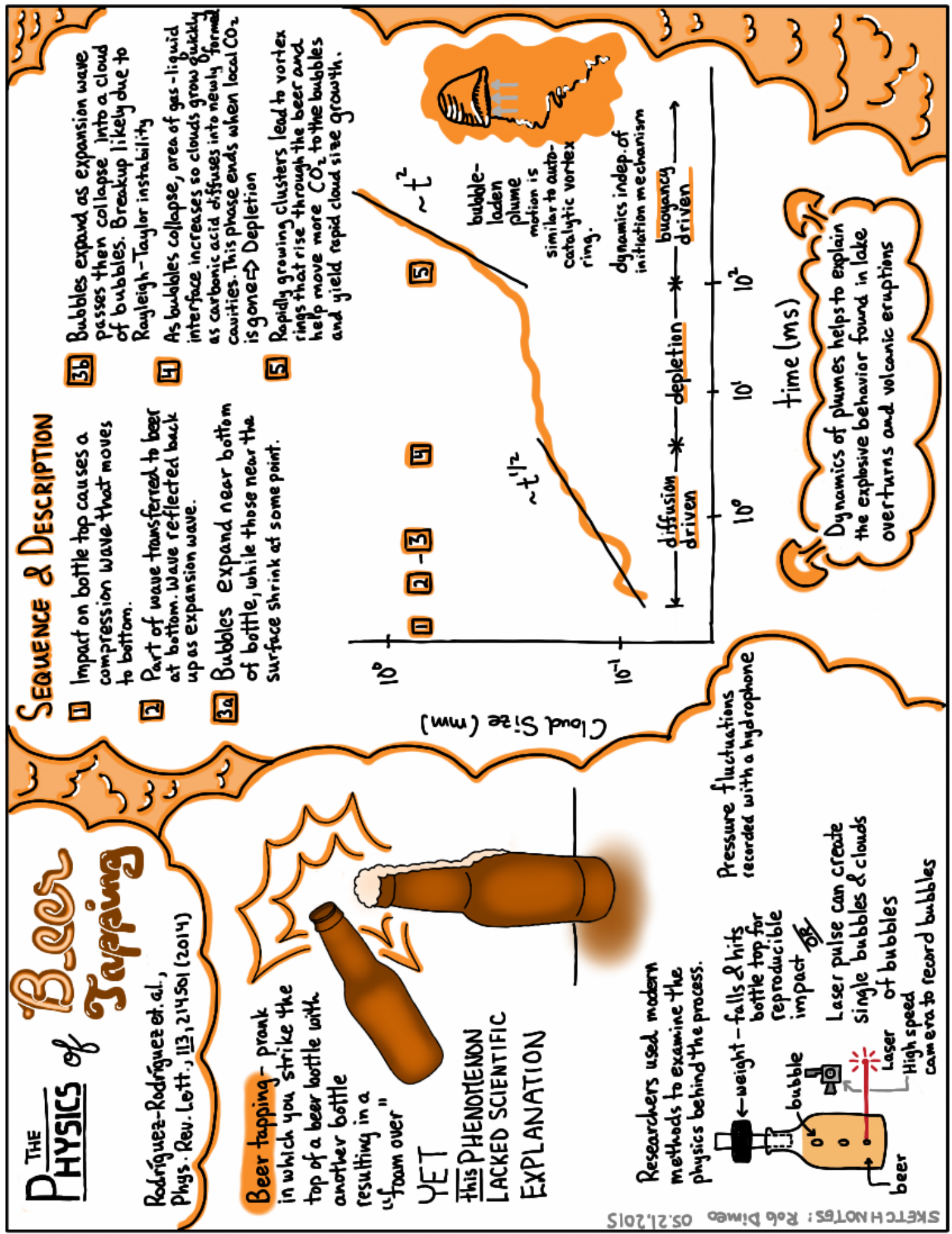

Fig. 5.3. Sketchnote based on the paper Physics of Beer Tapping[32]. 


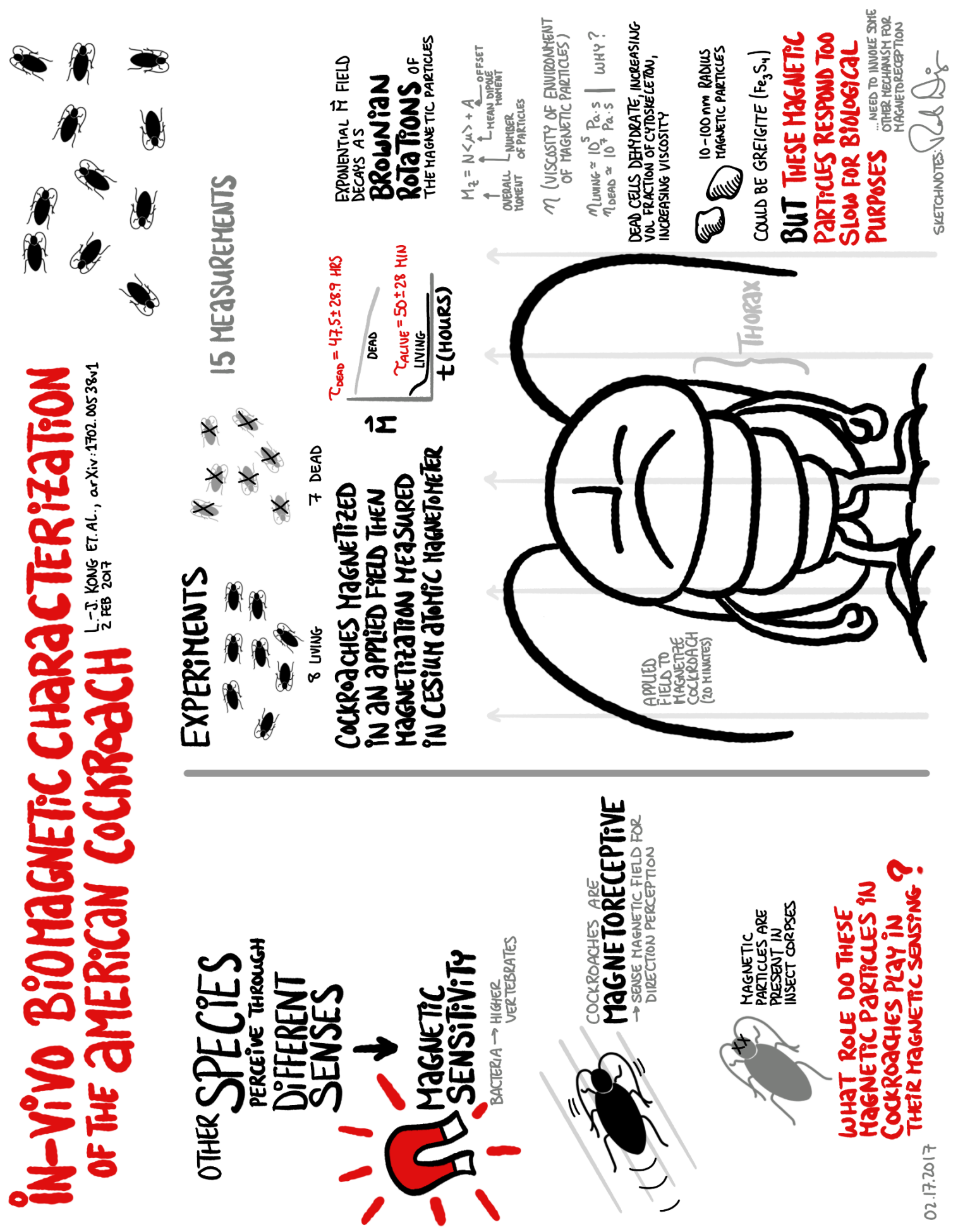

Fig. 5.4. Sketchnote based on the paper In-vivo biomagnetic characterisation of the American cockroach[10]. 
5 Selected Science Sketchnotes

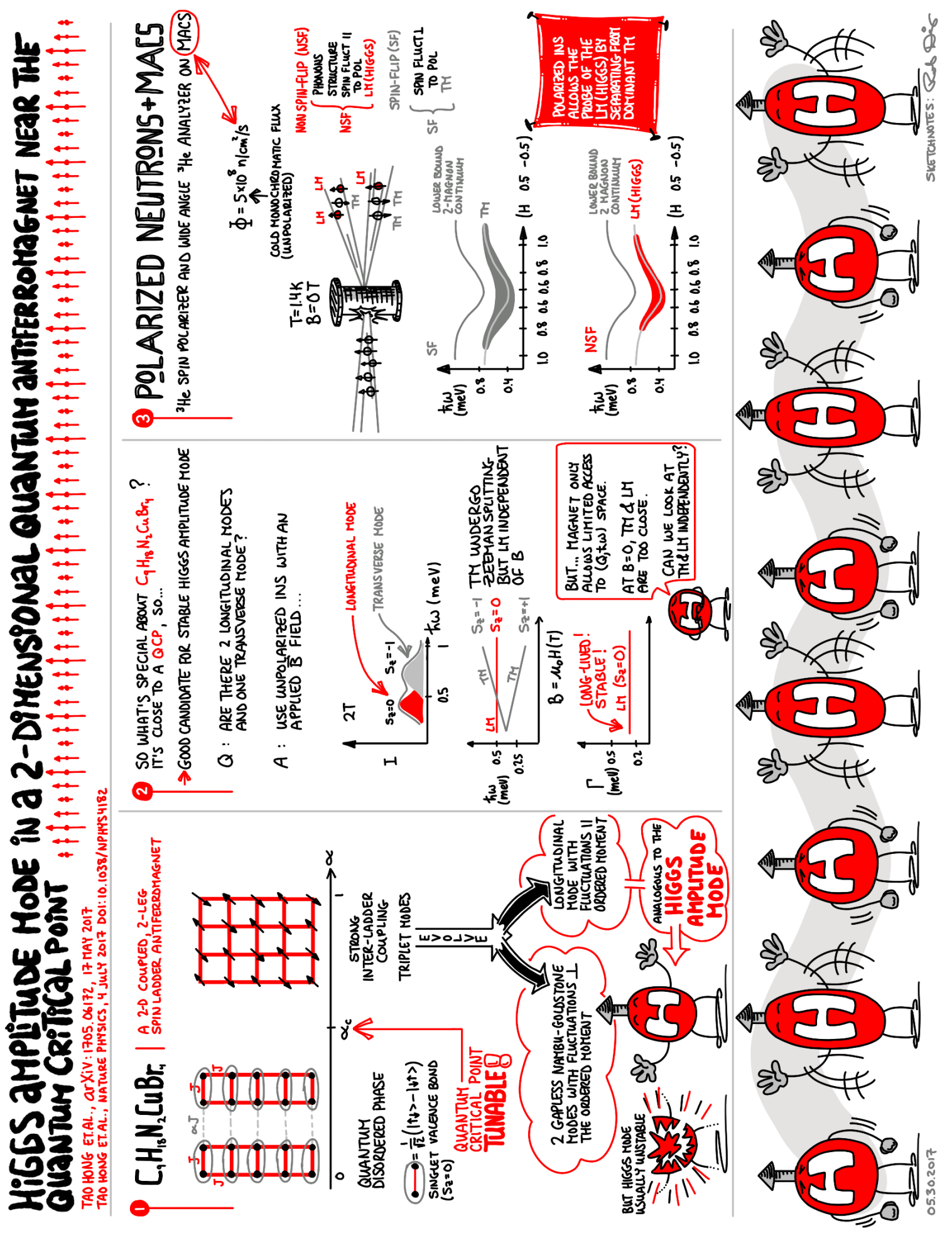

Fig. 5.5. Sketchnote based on the paper Higgs amplitude mode in a two-dimensional quantum antiferromagnet near the quantum critical point [33]. 


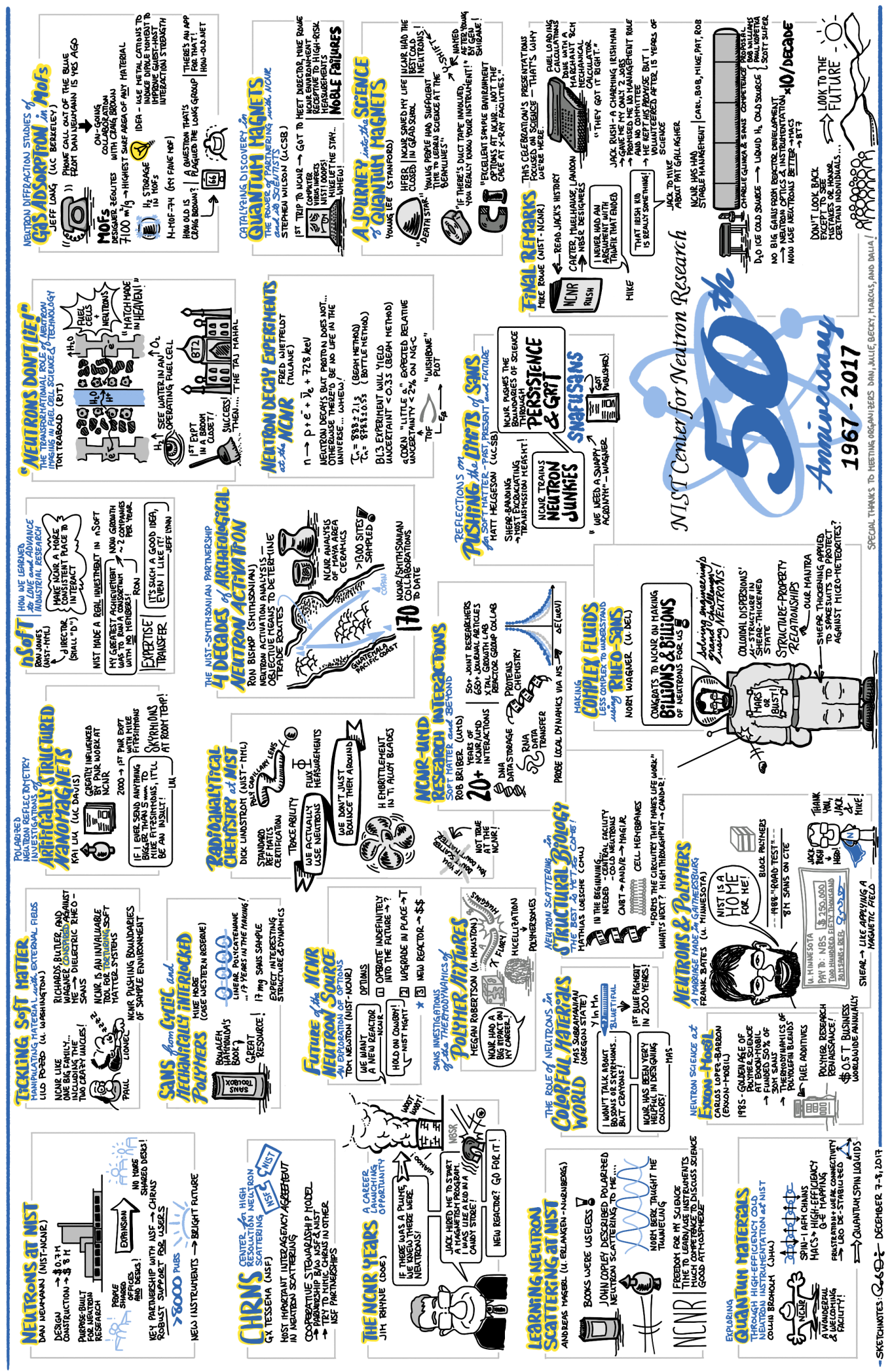

Fig. 5.6. Sketchnote based on numerous talks delivered at a technical symposium commemorating the 50-year anniversary of the NIST Center for Neutron Research. 
5 Selected Science Sketchnotes

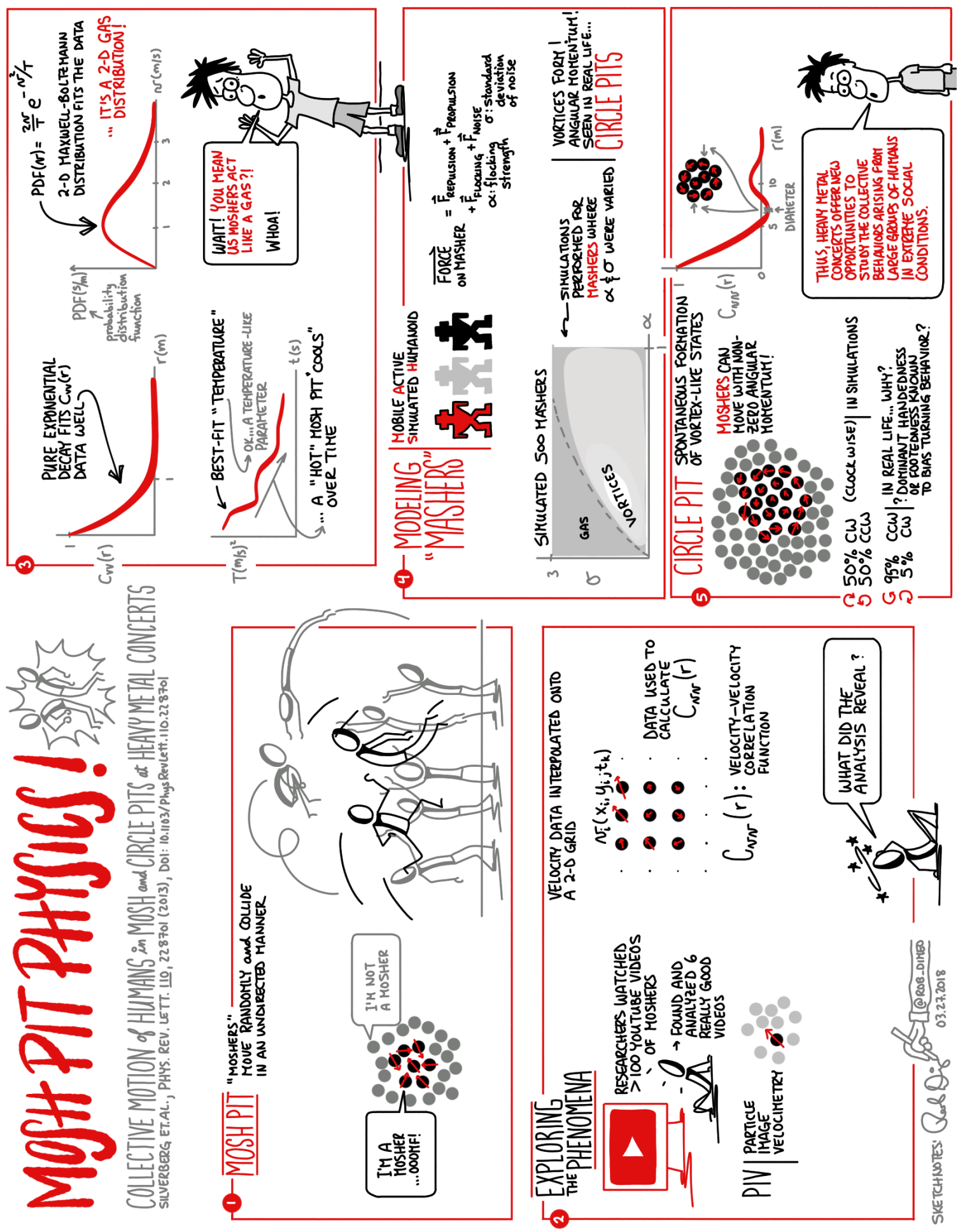

Fig. 5.7. Sketchnote based on the paper Collective Motion of Humans in Mosh and Circle Pits at Heavy Metal Concerts[4]. 


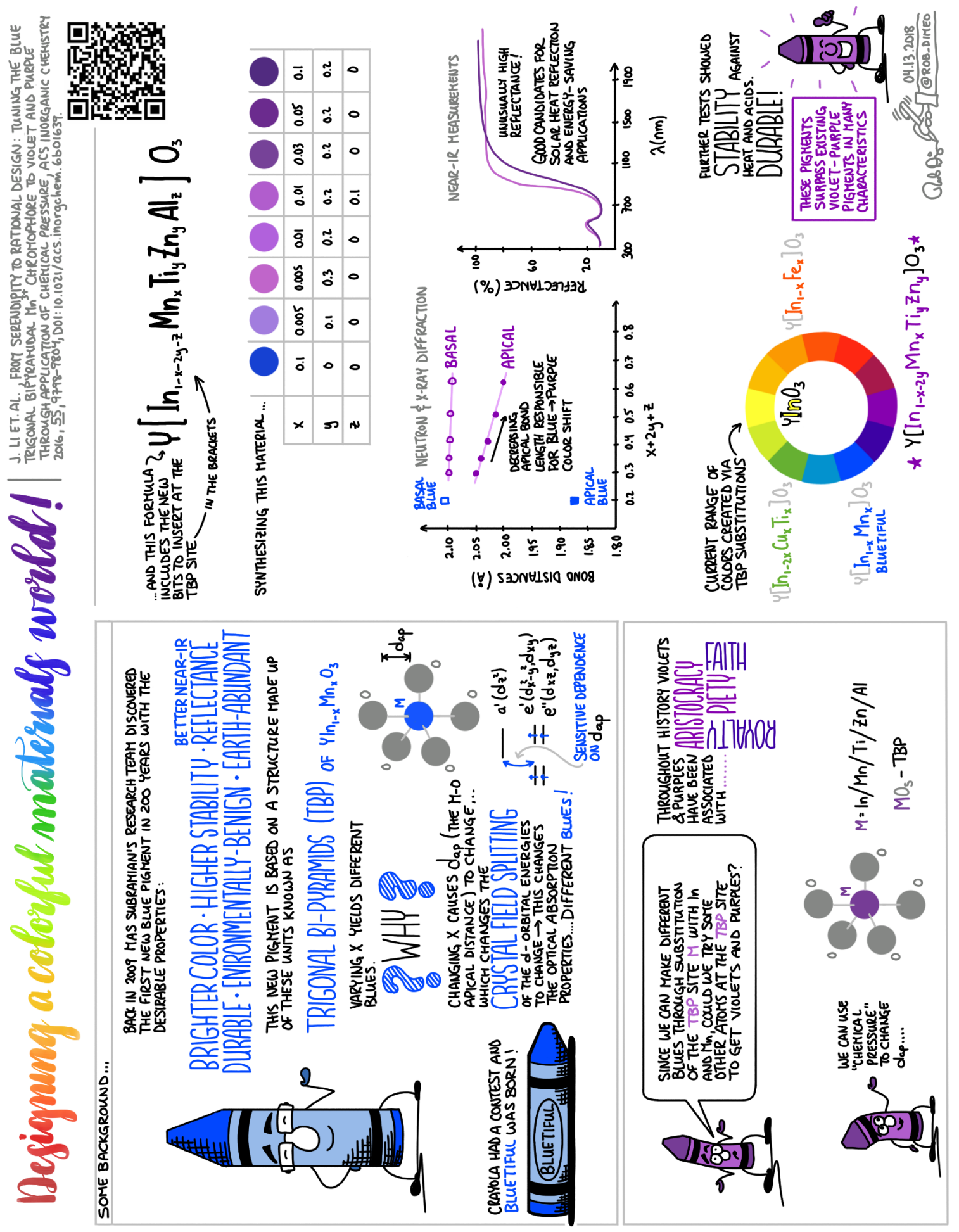

Fig. 5.8. Sketchnote based on the paper From Serendipity to Rational Design: Tuning the Blue Trigonal Bipyramidal $\mathrm{Mn}^{3+}$ Chromophore to Violet and Purple through Application of Chemical Pressure[34]. 
5 Selected Science Sketchnotes

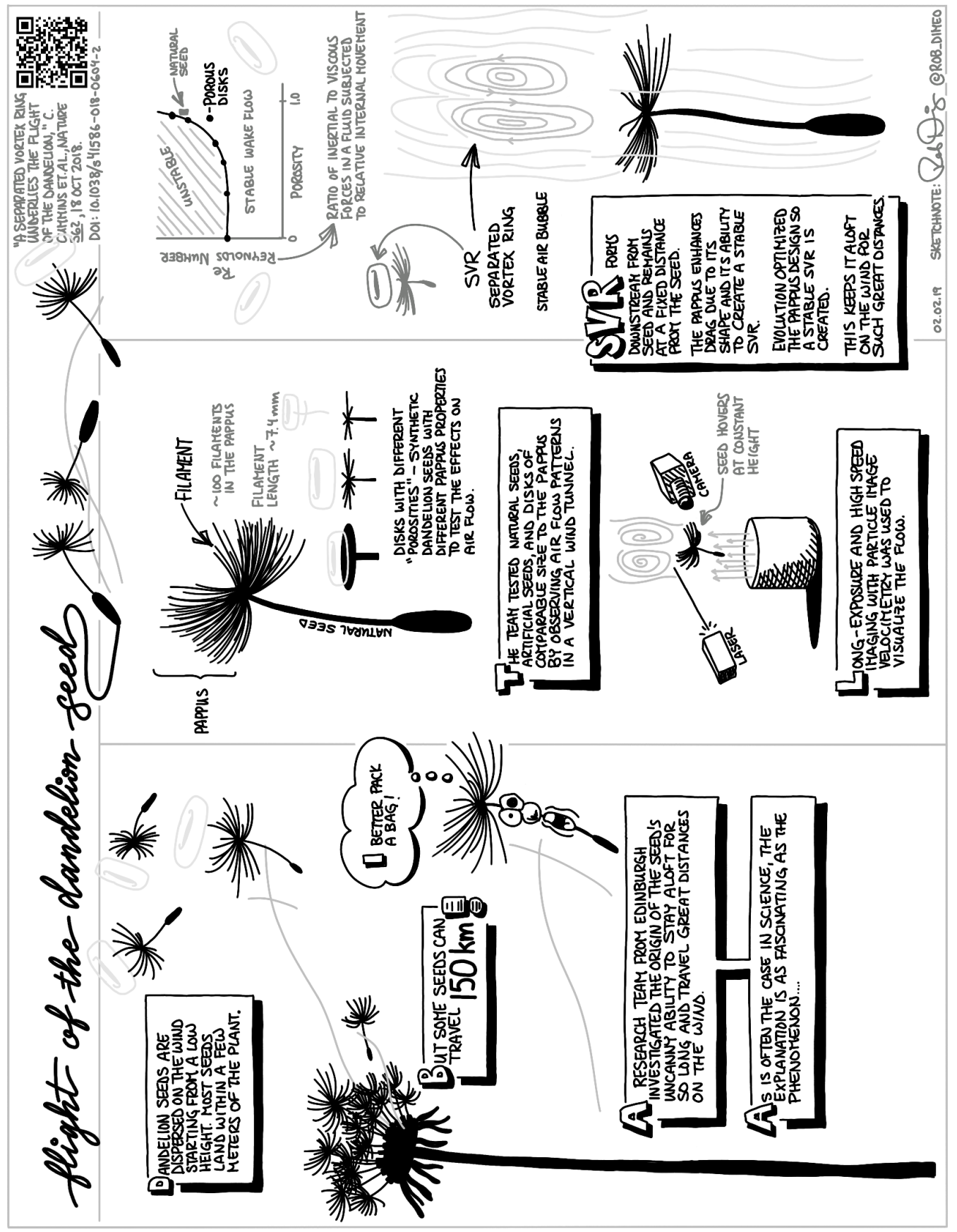

Fig. 5.9. Sketchnote based on the paper A separated vortex ring underlies the flight of the dandelion[11]. 


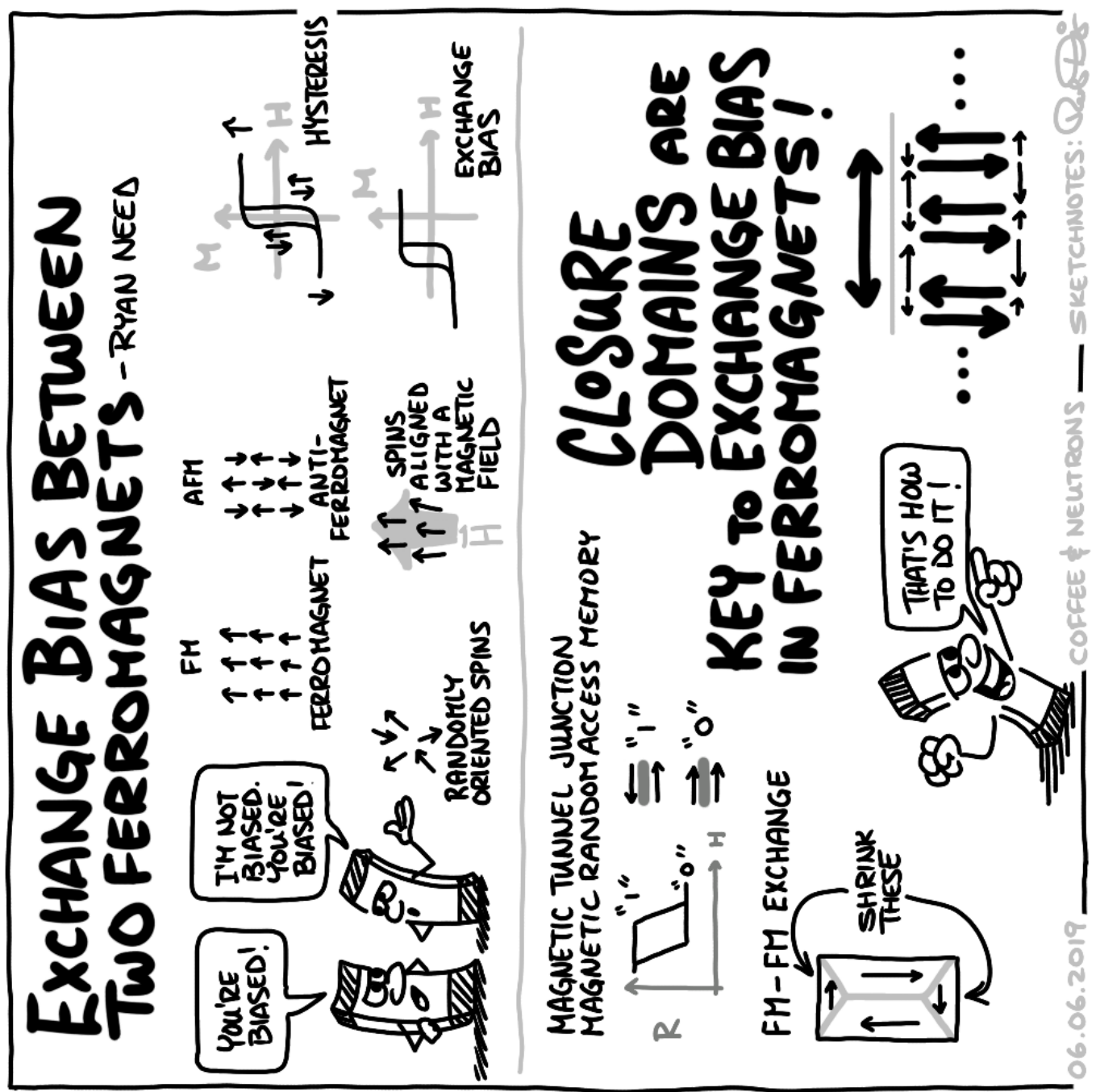

Fig. 5.10. Sketchnote based on a 10-minute Coffee \& Neutrons talk by Ryan Need at the NIST Center for Neutron Research on June 6, 2019. 


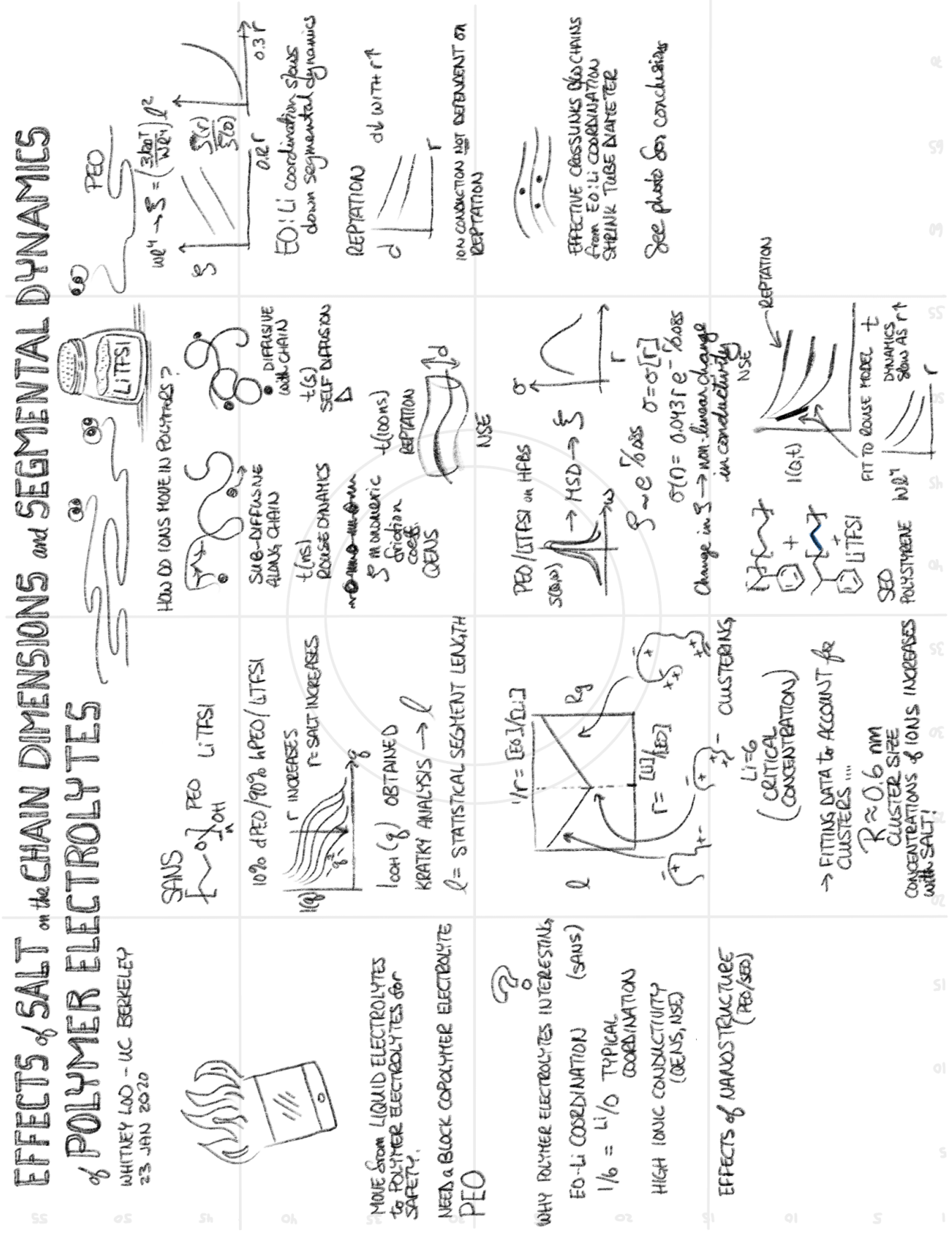

Fig. 5.11. Rough sketchnote based on a seminar at the NIST Center for Neutron Research by UC Berkeley's Whitney Loo on January 23, 2020. 


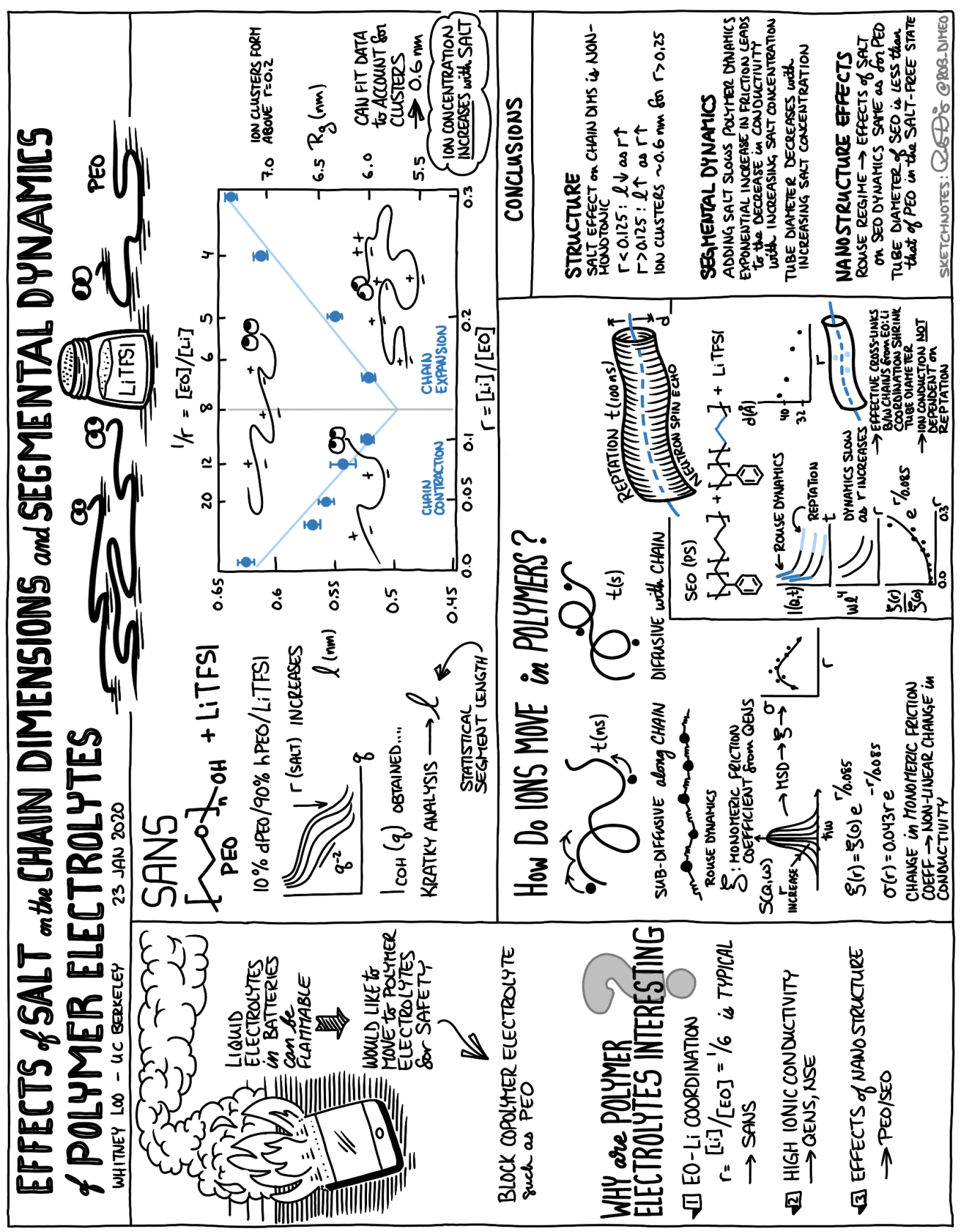

Fig. 5.12. Final sketchnote based on a seminar at the NIST Center for Neutron Research by UC Berkeley's Whitney Loo on January 23, 2020. 



\section{References}

[1] Rohde M (2013) The Sketchnote Handbook (Peachpit Press).

[2] Rohde M (2014) The Sketchnote Workbook (Peachpit Press).

[3] Pekar H (1986) The Young Crumb Story. American Splendor and More American Splendor: The Life and Times of Harvey Pekar (Peachpit Press, Doubleday/Dolphin), 1 st Ed.

[4] Silverberg JL, Bierbaum M, Sethna JP, Cohen I (2013) Collective motion of humans in mosh and circle pits at heavy metal concerts. Physical Review Letters 110(22):228701. https://doi.org/10.1103/PhysRevLett.110.228701

[5] Brown S (2014) The Doodle Revolution: Unlock the Power to Think Differently (Portfolio/Penguin).

[6] Wammes JD, Meade ME, Fernandes MA (2016) The drawing effect: Evidence for reliable and robust memory benefits in free recall. Quarterly Journal of Experimental Psychology 69(9):1752-1776. https://doi.org/10.1080/17470218.2015.1094494

[7] Mills E (2019) The Art of Visual Notetaking: An Interactive Guide to Visual Communication and Sketchnoting (Quarto Publishing Group).

[8] Ruben A (2016) How to read a scientific paper. Science https://doi.org/10.1126/ science.caredit.a1600012

[9] Einstein A (1956) On the movement of small particles suspended in a stationary liquid demanded by the molecular-kinetic theory of heat. Investigations on the Theory of the Brownian Movement (Dover Publications).

[10] Kong LJ, Crepaz H, Górecka A, Urbanek A, Dumke R, Paterek T (2018) In-vivo biomagnetic characterisation of the american cockroach. Scientific Reports 8(11):5140. https://doi.org/10.1038/s41598-018-23005-1

[11] Cummins C, Seale M, Macente A, Certini D, Mastropaolo E, Viola IM, Nakayama $\mathrm{N}$ (2018) A separated vortex ring underlies the flight of the dandelion. Nature 562:414-418. https://doi.org/10.1038/s41586-018-0604-2

[12] Quinn PV, Hong DC, Both JA (2005) Increasing the size of a piece of popcorn. Physica A: Statistical Mechanics and its Applications 353:637-648. https://doi.org/ 10.1016/j.physa.2005.02.013

[13] Virot E, Ponomarenko A (2015) Popcorn: critical temperature, jump and sound. Journal of The Royal Society Interface 12(104):20141247. https://doi.org/10.1098/rsif. 2014.1247

[14] Abel J, Madden M (2008) Drawing Words \& Writing Pictures: A Definitive Course 
from Concept to Comic in 15 Lessons (First Second) 1st Ed.

[15] Blitz B (1998) The Big Book of Cartooning (Courage Books).

[16] Pain E (2016) How to (seriously) read a scientific paper. Science https://doi.org/10. 1126/science.caredit.a1600047

[17] Morampudi SC, Turner AM, Pollmann F, Wilczek F (2017) Statistics of fractionalized excitations through threshold spectroscopy. Physical Review Letters 118(22):227201. https://doi.org/10.1103/PhysRevLett.118.227201

[18] Wilczek F (1982) Quantum mechanics of fractional-spin particles. Physical Review Letters 49(14):957-959. https://doi.org/10.1103/PhysRevLett.49.957

[19] Wilczek F (1982) Magnetic flux, angular momentum, and statistics. Physical Review Letters 48(17):1144-1146. https://doi.org/10.1103/PhysRevLett.48.1144

[20] Acharya R, Swamy PN (1994) Statistical mechanics of anyons. Journal of Physics A: Mathematical and General 27(22):7247-7263. https://doi.org/10.1088/0305-4470/ 27/22/005

[21] Gonick L, Criddle C (2005) The Cartoon Guide to Chemistry (Harper Collins).

[22] Chao LS, Schlamminger S, Newell DB, Pratt JR, Seifert F, Zhang X, Sineriz G, Liu M, Haddad D (2015) A LEGO watt balance: An apparatus to determine a mass based on the new SI. American Journal of Physics 83(11):913-922. https://doi.org/10.1119/ 1.4929898

[23] Mermin ND (1992) What's wrong with those talks? Physics Today 45(11):9. https://doi.org/10.1063/1.2809861

[24] Jamison A (2020) Ultracold molecules: From quantum chemistry to quantum computing. Perimeter Institute for Theoretical Physics Colloquium(20050010). Available at https://www.perimeterinstitute.ca/videos/ ultracold-molecules-quantum-chemistry-quantum-computing.

[25] Son H, Park JJ, Ketterle W, Jamison AO (2020) Collisional cooling of ultracold molecules. Nature 580:197-200. https://doi.org/10.1038/s41586-020-2141-z

[26] Mtangi W, Tassinari F, Vankayala K, Vargas Jentzsch A, Adelizzi B, Palmans ARA, Fontanesi C, Meijer EW, Naaman R (2017) Control of electrons' spin eliminates hydrogen peroxide formation during water splitting. Journal of the American Chemical Society 139(7):2794-2798. https://doi.org/10.1021/jacs.6b12971

[27] Roam D (2016) Draw to Win: A Crash Course on How to Lead, Sell, and Innovate With Your Visual Mind (Portfolio).

[28] Dimeo R (2016) Sketchnoting: An analog skill in the digital age. ACM SIGCAS Computers and Society 46(3):9-16. https://doi.org/10.1145/3024949.3024951

[29] Roam D (2014) Show \& Tell: How Everybody Can Make Extraordinary Presentations (Portfolio/Penguin).

[30] Dimeo R (2019) Instrument design and innovation at the NIST Center for Neutron Research. 8th annual Design and Engineering of Neutron Instruments Meeting (Bethesda, MD). Available at https://denim2019.umd.edu/

[31] Dimeo R (2016) Neutron backscattering at NIST. 50 Years of Neutron Backscattering Spectroscopy (Garching, Germany). Available at https://indico.frm2.tum.de/event/30/ 
[32] Rodríguez-Rodríguez J, Casado-Chacón A, Fuster D (2014) Physics of beer tapping. Physical Review Letters 113(21):214501. https://doi.org/10.1103/PhysRevLett.113. 214501

[33] Hong T, Matsumoto M, Qiu Y, Chen W, Gentile TR, Watson S, Awwadi FF, Turnbull MM, Dissanayake SE, Agrawal H, et al (2017) Higgs amplitude mode in a twodimensional quantum antiferromagnet near the quantum critical point. Nature Physics 13(7):638-642. https://doi.org/10.1038/nphys4182

[34] Li J, Lorger S, Stalick JK, Sleight AW, Subramanian MA (2016) From serendipity to rational design: Tuning the blue trigonal bipyramidal mn3+ chromophore to violet and purple through application of chemical pressure. Inorganic Chemistry 55(19):9798-9804. https://doi.org/10.1021/acs.inorgchem.6b01639 